\title{
A particle method for two-phase flows with compressible air pocket
}

\author{
M. Luo*, C. G. Koh, W. Bai and M. Gao \\ Department of Civil and Environmental Engineering, National University of Singapore, Singapore 117576
}

\begin{abstract}
SUMMARY
When using particle methods to simulate water-air flows with compressible air pockets, a major challenge is to deal with the large differences in physical properties (e.g. density and viscosity) between water and air. In addition, the accurate modelling of air compressibility is essential. To this end, a new two-phase strategy is proposed to simulate incompressible and compressible fluids simultaneously without iterations between the solvers for incompressible and compressible flows. Water is modeled by the recently developed 2-phase Consistent Particle Method (2P-CPM) for incompressible flows. For air modeling, a new compressible solver is proposed based on the ideal gas law and thermodynamics. The formulation avoids the problem of determining the actual sound speed which is dependent on the temperature and is therefore not necessarily constant. In addition, the compressible air solver is seamlessly integrated with the incompressible solver 2P-CPM because they both use the same predictor-corrector scheme to solve the governing equations. The performance of the proposed method is demonstrated by three benchmark problems as well as an experimental study of sloshing impact with entrapped air pockets in an oscillating tank.
\end{abstract}

Keywords: compressibility; two-phase flow; air pocket; polytropic gas law; particle method

\section{INTRODUCTION}

Most two-phase flow models [1-3] treat both gas and liquid as incompressible. While incompressibility is a reasonable assumption in some water-air flow scenarios [4], air entrapment or entrainment may be generated in some other problems such as violent wave impact on structures [5, 6]. The compressibility of entrapped air pockets can play an important role in the water-air interaction in terms of influencing the pressure peak and impact duration in a wave impact process $[7,8]$. Therefore, it is necessary to include air compressibility to better simulate such water-air flow problems.

The main numerical difficulties to model water-air flows with entrapped air pockets include the large and discontinuous deformation of fluid and the abrupt discontinuity of fluid properties (density and viscosity) at the interface between water and air. An even greater challenge is to have an

\footnotetext{
${ }^{*}$ Corresponding author. Tel.: +6565162163 ; fax: +6567791635.

E-mail address: luomin@u.nus.edu; cveluom@163.com
} 
integrated solution for water and air that behave very differently, the former being practically incompressible and the latter highly compressible. To address these issues, many mesh-based methods (such as Finite Difference Method and Finite Volume Method) and meshless methods have been developed. While Element Free Galerkin Method [9] and Particle Finite Element Method [10] may be considered "meshless" methods, they still require mesh to define shape functions with which the governing equations are solved, although the particles (nodes) can move independently of the mesh. The main meshless methods that completely remove the need of mesh include Smoothed Particle Hydrodynamics (SPH), Incompressible Smoothed Particle Hydrodynamics (ISPH) and Moving Particle Semi-implicit (MPS) method. Due to the meshless and Lagrangian nature, particle methods possess three inherent advantages over mesh-based methods: (1) better capability in modeling large and discontinuous fluid motion such as breaking waves, (2) better tracking of moving interface of different fluids, and (3) no numerical diffusion induced by the convection term in the Navier-Stokes equation. Considering these advantages, a particle method is adopted in this study.

The main differences between the three commonly used particles methods, i.e. SPH, ISPH and MPS, lie in the computations of fluid pressure and spatial derivatives (for gradient and Laplacian). In $\mathrm{SPH}$, the fluid pressure is explicitly solved by an equation of state (EOS), whereas ISPH computes fluid pressure implicitly by solving a pressure Poisson equation (PPE). Both SPH and ISPH adopt the same derivative-approximation schemes which essentially use a kernel function (with finite values in a finite influence region) to approximate Dirac delta function. The underlying principle is that, in the limiting case as the influence radius approaches zero, the kernel function would approach the Dirac delta function. Nevertheless, since the influence radius should be sufficiently large to involve sufficient neighbor particles for computation, it would be difficult for any kernel function to accurately approximate Dirac delta function which has an infinitely high value over an infinitesimal region. While a good choice of kernel function may be appropriate to approximate a scalar variable, its use in computing spatial derivatives would require the derivative of a kernel function, which is tantamount to approximating the (non-existent) derivative of Dirac delta function. Similar to ISPH, MPS solves fluid pressure implicitly by solving PPE but using a different derivative approximation strategy. The gradient term is computed as a weighted average of the gradient vectors evaluated between the reference particle and all neighbor particles. Derived based on the analogy of transient diffusion, the Laplacian operator of a parameter is a weighted average of the quantities distributed from neighbor particles.

In computing derivatives, all these methods need a predefined kernel or weighting function, which is non-unique and has significant influence on the numerical accuracy. Particularly when the particle distribution is irregular (inevitable in violent fluid motion), large numerical errors would be generated and the inaccurate approximation of derivatives would induce spurious pressure fluctuation. 
Consequently, numerical treatments such as artificial viscosity, artificial sound speed and artificial surface tension are required in some of these methods on free-surface or two-phase flow problems.

To address this issue, a new meshless method named Consistent Particle Method (CPM) has recently been proposed [11]. In this method, the spatial derivatives are solved by adopting the Generalized Finite Difference (GFD) scheme [12]. Being consistent with Taylor series expansion and eliminating the use of a kernel function, CPM is fundamentally different from the above-mentioned particle methods in terms of the derivative-approximation scheme. Note also that the first-order derivatives (for gradient) and second-order derivatives (for Laplace operator) are simultaneously solved instead of using different equations unlike some other particle methods. Due to the accurate computation of derivatives, the problem of spurious pressure fluctuation that exists in some other particle methods has been largely resolved $[11,13]$. This also enhances computational efficiency since, with more accurate pressure solution, an iterative solver would be able to converge fast when solving the PPE. More specifically, the computational efficiency of CPM should be at least comparable to those of MPS and ISPH, both of which need to solve a PPE [14]. Considering the advantages of CPM including its consistent computation of spatial derivatives and smoother pressure solution, this method has been recently extended to model incompressible two-phase flows with large density difference [14]. This involves a new derivative-computation scheme accounting for abrupt density discontinuity and an adaptive particle selection scheme. The method is able to model violent water-air flows with density ratio of about a thousand between the two phases. Nevertheless, for problems where compression of air is significant, it would be necessary to account for air compressibility while treating water as incompressible. Therefore, the incompressible 2P-CPM [14] is further improved by accounting for air compressibility in the present study such that water-air flows with air entrapment can be accurately simulated.

To model incompressible or weakly compressible flows, the approach of artificial compressibility was used by other researchers $[4,15]$, by replacing the transient density term in the continuity term with a pressure term through an EOS. Nevertheless, employing the real compressibility parameter (via sound speed $c_{s}$ ) may require very small time step to satisfy the Courant-Friedrichs-Lewy (CFL) condition [16]. Therefore, an artificial value of sound speed (generally much smaller than the real value) is adopted in many studies; otherwise the required time step would be very small. However, since fluid compressibility is related to the sound speed, the choice of artificial sound speed would affect the accuracy of the compressible solver significantly [17]. Hence, careful calibration is required to achieve a compromise between accuracy and efficiency for each case. While most of the artificial compressibility studies are mesh based, some particle methods such as SPH also use a similar concept to simulate water-air two-phase flows [18]. The compressibility of air and water is controlled by different artificial sound speeds which are often very different from the actual values. 
Besides artificial compressibility, the predictor-corrector (or split) scheme has been used to model compressible flows. Such studies include the C-CUP (C for Cubic-interpolated propagation and CUP for combined unified procedure) [19] and its variants [20,21]. Due to the implicit treatment of fluid pressure, a larger time step can be used and the computed pressure is generally more stable than the artificial compressibility approach $[16,20]$. In addition, these methods can be readily formulated to model incompressible and compressible fluids simultaneously because they solve the Navier-Stokes equations of primitive (non-conservative) form [21]. Based on the predict-corrector scheme, MPS has also been used for compressible flows by using the sound speed dependent EOS. Using this compressibility scheme, Ikari et al. [22] presented a decoupled MPS model in which the incompressible water and compressible air domains were solved separately and the interaction simulated by force terms. Even though relatively stable results were obtained, this decoupled scheme could not accurately model the interaction of different fluids near interface accurately. From the viewpoints of formulation elegance and ease of implementation, the same computational procedure to couple incompressible water and compressible air is desired.

To model compressible flow, the most commonly used EOS involves sound speed which is given by $c_{s}=\sqrt{\partial p / \partial \rho}$ where $p$ and $\rho$ are the fluid pressure and density respectively. An advantage of this type of EOS is that it can describe both liquid and gas. Nevertheless, when modelling violent waterair flows, this approach has some limitations because $c_{s}$ is dependent on the composition [23] and temperature of fluid [24]. Therefore, it is difficult to determine the correct value of sound speed in different scenarios (e.g. during and immediately after wave impact in a wave impact problem).

Another type of EOS for compressible gases is the ideal gas law, i.e. $p=\rho R T$, where $T$ is temperature and $R$ is the gas constant. The ideal gas law can be written into the form $p=(\gamma-1) \rho e$, where $\gamma$ is the ratio of specific heats and $e$ the internal energy [25]. The ideal gas law governs many real gases of engineering interests under normal pressure and temperature. By assuming an isentropic process, the ideal gas law leads to the polytropic gas law $p_{2} / p_{1}=\left(\rho_{2} / \rho_{1}\right)^{\gamma}$. Because of its simplicity, this type of EOS has been used to model air pockets or bubbles $[5,26,27]$. Compared to the $c_{s}$ dependent EOS, the polytropic formulation does not involve the sound speed and thus avoids the issue of determining the real (or artificial) sound speed. In some studies [5, 26, 27], however, the air pressure is assumed to be spatially uniform in each air pocket and hence the spatial variation of air pressure (e.g. pressure wave) could not be captured.

In this study, a new compressible solver is proposed that is consistent with thermodynamics. This solver does not involve sound speed nor assume uniform pressure in the entrapped air pocket. In addition, based on the same predictor-corrector scheme, this compressible solver can be coupled seamlessly with the recently developed incompressible solver for two-phase flows [14]. This leads to 
the complete 2P-CPM capable of simulating two-phase incompressible and compressible flows with a wide range of density ratio. Using the proposed method, wave-impact problems in which air compressibility is important are studied. In addition to comparison with published results, an experimental study of water sloshing with air pocket in an oscillating task is conducted for validation purpose.

\section{CONSISTENT PARTICLE METHOD}

The governing equations for viscous Newtonian fluids (both incompressible and compressible) in a two-fluid system are the Navier-Stokes equations as follows [28]:

$$
\begin{gathered}
\frac{1}{\rho} \frac{D \rho}{D t}+\nabla \cdot \mathbf{v}=0 \\
\frac{D \mathbf{v}}{D t}=-\frac{1}{\rho} \nabla p+\frac{1}{\rho} \nabla \cdot\left[\mu\left(\nabla \mathbf{v}+(\nabla \mathbf{v})^{T}\right)\right]+\mathbf{g}
\end{gathered}
$$

where $\rho$ is the density of fluid, $\mathbf{V}$ the particle velocity vector, $p$ the fluid pressure, $\mu$ the dynamic viscosity of fluid and $\mathbf{g}$ the gravitational acceleration. The unified equations for incompressible and compressible fluids make it possible to model flows of different phases simultaneously.

For both incompressible and compressible fluids, Equations (1) and (2) can be solved by a predictor-corrector scheme. In the predictor step, the intermediate velocity $\mathbf{v}^{*}$ and position $\mathbf{r}^{*}$ of fluid particles are computed by neglecting the pressure gradient term in Equation (2) as

$$
\begin{gathered}
\mathbf{v}^{*}=\mathbf{v}^{(k)}+\left\{\frac{1}{\rho} \nabla \cdot\left[\mu\left(\nabla \mathbf{v}^{(k)}+\left(\nabla \mathbf{v}^{(k)}\right)^{T}\right)\right]+\mathbf{g}\right\} \Delta t \\
\mathbf{r}^{*}=\mathbf{r}^{(k)}+\mathbf{v}^{*} \Delta t
\end{gathered}
$$

where $\Delta t=t^{(k+1)}-t^{(k)}, \mathbf{v}^{(k)}=\mathbf{v}\left(\mathbf{r}^{(k)}, t^{(k)}\right), \mathbf{v}^{(k)}$ and $\mathbf{r}^{(k)}$ are the particle velocity and position at time $t^{(k)}$.

In the corrector step, a PPE can be derived as follows:

$$
\nabla \cdot\left(\frac{1}{\rho^{*}} \nabla p^{(k+1)}\right)=\frac{1}{\Delta t^{2}} \frac{\rho^{(k+1)}-\rho^{*}}{\rho^{(k+1)}}
$$

For incompressible fluids, the incompressibility condition $\rho^{(k+1)}=\rho_{0}$, where $\rho_{0}$ is the initial fluid density, is imposed on the right-hand side of Equation (5). Within the influence radius $r_{e}\left(r_{e}=3.1 L_{0}\right.$ is adopted in one-dimensional cases and $r_{e}=2.1 L_{0}$ is adopted in two-dimensional cases of the present 
study to achieve a balance between accuracy and efficiency, where $L_{0}$ is the initial particle spacing), the intermediate fluid density $\rho^{*}$ for particle $i$ is evaluated as [29]

$$
\rho_{i}^{*}=m_{i} \sum_{j} w\left(r_{i j}\right)
$$

where $r_{i j}$ is the distance between the reference particle $(i)$ and neighbor particles $(j)$ based on the intermediate positions, $w\left(r_{i j}\right)$ a weighting function defined in [13], and $m_{i}$ the mass of particle $i$ (fixed during simulation). Letting the initial density of particle $i$ be $\rho_{0 i}, m_{i}$ can be computed based on the initial particle distribution as follows:

$$
m_{i}=\rho_{0 i} / \sum_{j} w\left(r_{i j}\right)
$$

For compressible fluids, the intermediate density is evaluated in the same way as incompressible fluids but with a different weighting function. As pointed out by Koshizuka and Oka [30], the sharp weighting function generates large repulsive force when particles get closer and hence is suitable in the simulation of incompressible flows. In contrast, the slow-slope weighting functions whose value at $r=0$ is smaller allows more compressibility of fluids, such as the following function [31]

$$
w_{2}\left(r_{i j}\right)=\left\{\begin{array}{cc}
1-6\left(\frac{r}{r_{e}}\right)^{2}+8\left(\frac{r}{r_{e}}\right)^{3}-3\left(\frac{r}{r_{e}}\right)^{4}, & 0 \leq r \leq r_{e} \\
0 \quad, r_{e}<r
\end{array}\right.
$$

Using Equation (8), the computed air density agrees well with the theoretical value when air is compressed or expanded. This is the first difference in modelling incompressible water and compressible air. Another distinct feature in the simulation of compressible flows is that, without the incompressibility condition, the fluid density $\rho^{(k+1)}$ in Equation (5) should be treated as unknown (more details will be presented in the following section).

Applying the derivative computation scheme as shown in Equation (13) [14] to the left hand side of Equation (5) and Equation (24) leads to a system of linear equations with sparse coefficients, which can be solved effectively by the generalized minimal residual (GMRES) method with incomplete LU factorization [32]. Using the solved fluid pressure, particle velocities and positions are updated as

$$
\mathbf{v}^{(k+1)}=\mathbf{v}^{*}-\left(\frac{\nabla p}{\rho}\right)^{(k+1)} \Delta t
$$

and

$$
\mathbf{r}^{(k+1)}=\mathbf{r}^{(k)}+\mathbf{v}^{(k+1)} \Delta t
$$


where the pressure gradient term is computed by Equation (12) [14] and the time step $\Delta t$ at step $k+1$ has to satisfy the Courant condition as [33]

$$
\Delta t \leq 0.2 \frac{L_{0}}{v_{\max }}
$$

where $v_{\max }$ is the maximum particle velocity at step $k$.

The above solution scheme is similar to those in MPS [34] and ISPH [33]. Nevertheless, there are two significant differences between the proposed 2P-CPM and other particle methods for two-phase flows. The first difference is that the proposed method computes spatial derivative (for gradient and Laplacian operators) for two fluids with drastic density difference (e.g. water versus air) on the basis of Taylor series expansion. The computation of first- and second order derivatives (in the $x$ direction) is as follows (more details are given in [11] and [14]):

$$
\left(\frac{1}{\rho} \frac{\partial p}{\partial x}\right)_{i}=\sum_{j \neq i}\left[\frac{1}{0.5\left(\rho_{i}+\rho_{j}\right)} C_{1 j}\left(p_{j}-p_{i}\right)\right]
$$

and

$$
\left(\frac{\partial}{\partial x}\left(\frac{1}{\rho} \frac{\partial p}{\partial x}\right)\right)_{i}=\sum_{j \neq i}\left[\frac{1}{0.5\left(\rho_{i}+\rho_{j}\right)} C_{3 j}\left(p_{j}-p_{i}\right)\right]
$$

where $\rho_{i}$ and $\rho_{j}$ are the fluid density at particle $i$ and $j, p_{i}$ and $p_{j}$ are the fluid pressure at particle $i$ and $j$, and $C_{1 j}$ and $C_{3 j}$ are the coefficients (dependent on the relative positions between the reference particle and its neighboring particles) generated by GFD. In a similar way, the derivatives along the $y$ direction are computed. Since the gradient and Laplacian terms on an interface particle (whose influence domain contains water and air particles) are computed by using the neighbor particles of both fluids and the whole computational domain (both water and air) is solved simultaneously, the interaction forces between interface particles can be accurately simulated. This is in contrast to some decoupled models for two-fluid or two-phase flows such as Ikari et al. [22] and Gotoh and Sakai [35] in which the interaction forces between interface particles were represented by force terms. Besides dealing with drastic density difference, CPM is able to treat abrupt viscosity change across fluid interface by enforcing the continuity condition of viscous stress at the interface (see Appendix A). This capability has been demonstrated in [14] with a Rayleigh-Taylor instability example in which fluid viscosity plays an important role. Note that the derivation of spatial derivatives of pressure and velocity in CPM involves imposing the continuity of fluid motion and traction forces at the two-fluid interface (Appendix A). 
The second significant feature of the present $2 \mathrm{P}-\mathrm{CPM}$ is that a thermodynamically consistent compressible solver is formulated to account for air compressibility, as elaborated below.

\section{COMPRESSIBLE SOLVER}

For incompressible flow (liquid), the fluid density at the current time step, i.e. $\rho^{(k+1)}$ in Equation (5), is enforced to be $\rho_{0}$. Therefore, the PPE with only one variable can be directly solved to obtain fluid pressure. For compressible flows (gas), however, $\rho^{(k+1)}$ is unknown and hence an additional condition called closure condition is needed to solve the pressure equation. The closure condition is obtained from the relation linking fluid properties in the context of thermodynamics. The main idea is briefly explained next.

\subsection{Thermodynamic considerations}

The pressure $p$, density $\rho$ (or volume $V$ ) and temperature $T$ are related by an EOS, the general form of which is

$$
f(p, \rho, T)=0
$$

Some useful relations can be derived [25], including the speed of sound as follows

$$
c_{s}=\sqrt{\left(\frac{\partial p}{\partial \rho}\right)_{s}}
$$

where subscript $s$ means that the derivative is taken at constant entropy.

As mentioned earlier, some studies used the above equation to relate fluid pressure and density. These studies $[4,18]$, however, have some drawbacks such as the need to determine numerical $c_{s}$ which is not necessarily the actual sound speed and has to be calibrated. To address this issue, the present study employs the polytropic gas law without using the speed of sound explicitly.

\subsection{Polytropic gas law}

At normal pressure and temperature, most real gases can be assumed to obey the ideal gas law as follows [36]:

$$
p=\rho R T
$$

Based on the First and Second Laws of Thermodynamics, and assuming an adiabatic and reversible (hence isentropic) process, the following 'polytropic' equation can be readily obtained

$$
\frac{p}{\rho^{\gamma}}=\text { constant }
$$


where $\gamma$ is the ratio of specific heats at constant pressure and constant volume. Its value for air is about 1.4. Equation (17) is also a special case of the more general polytropic process governed by $p / \rho^{n}=$ constant, where $n$ is called the polytropic index and $n=\gamma$ for isentropic processes.

In real applications such as wave impact problems in marine and offshore engineering, the wave impact process is generally very rapid. There is little time for heat transfer to take place significantly between an air pocket and its surroundings such as water particles (and solid boundary if any) that entrap the air particles. Hence, it is reasonable to assume adiabatic process for air in wave-impact problems (the main focus of this paper). In addition, since energy loss is small due to the low viscosity of air, the air expansion or compression process in two-phase flow problems can be approximated as reversible. With these two assumptions, Equation (17) with $n=\gamma$ is applicable and will be utilized in the present study unless otherwise stated. This value has also been adopted in other numerical studies on wave impact problems $[5,26,27]$ and verified experimentally by Abrahamsen and Faltinsen $[8]$ in their work of sloshing impact on tank walls.

Derived in the thermodynamics framework, the polytropic gas law for ideal gases does not contain the parameter of $c_{s}$. Hence it avoids the problem to determine sound speed (dependent on the composition and temperature of a fluid) in numerical simulation, unlike in the $c_{s}$ dependent EOS. Therefore, the polytropic gas law is used as the constitutive equation for modeling air compressibility in two-phase flow problems.

\subsection{Pressure Poisson equation considering fluid compressibility}

Choosing a reference state, the polytropic gas law can be written as

$$
\rho=\rho_{\mathrm{ref}} \frac{p^{1 / \gamma}}{p_{\mathrm{ref}}^{1 / \gamma}}
$$

where $\rho_{\text {ref }}$ and $p_{\text {ref }}$ are the gas density and pressure at the reference state. There are two options for the reference state: (a) the initial state and (b) the previous time step. The latter option has been shown to induce the propagation of numerical errors [24]. Therefore, the initial values, i.e. $\rho_{a 0}$ and $p_{a 0}$, are adopted herein as the reference values.

Replacing $\rho_{\text {ref }}$ and $p_{\text {ref }}$ by $\rho_{a 0}$ and $p_{a 0}$ in Equation (18) and taking derivative with respect to $p$ yields

$$
\frac{d \rho}{d p}=\frac{\rho_{a 0}}{p_{a 0}^{1 / \gamma}} \frac{1}{\gamma} p^{1 / \gamma-1}
$$

By Taylor series expansion, the air density at time step $k+1$, i.e. $\rho^{(k+1)}$, can be approximated as 


$$
\rho^{(k+1)}=\rho_{a 0}+\left.\frac{d \rho}{d p}\right|_{p=p_{a 0}} \cdot\left(p^{(k+1)}-p_{a 0}\right)
$$

Substituting Equation (19) into (20) gives

$$
\rho^{(k+1)}=\rho_{a 0}+\frac{\rho_{a 0}}{p_{a 0}} \frac{1}{\gamma} \cdot\left(p^{(k+1)}-p_{a 0}\right)
$$

To incorporate this relationship in the PPE, the right hand side of Equation (5) is split as

$$
\nabla \cdot\left(\frac{1}{\rho^{*}} \nabla p^{(k+1)}\right)=\frac{1}{\Delta t^{2}} \frac{\rho_{a 0}-\rho^{*}+\rho^{(k+1)}-\rho_{a 0}}{\rho^{(k+1)}}
$$

Since $\rho^{(k+1)}$ also exists in the denominator, the right hand side of Equation (22) is a non-linear term. To linearize it, $\rho^{(k+1)}$ is approximated as $\rho^{*}$ because the difference between the intermediate and corrected fluid densities is small in each sufficiently small time step, leading to

$$
\nabla \cdot\left(\frac{1}{\rho^{*}} \nabla p^{(k+1)}\right)=\frac{1}{\Delta t^{2}} \frac{\rho_{a 0}-\rho^{*}}{\rho^{*}}+\frac{1}{\Delta t^{2}} \frac{\rho^{(k+1)}-\rho_{a 0}}{\rho^{*}}
$$

Substituting Equation (21) into (23), the pressure equation accounting for fluid compressibility is

$$
-\nabla \cdot\left(\frac{1}{\rho_{i}^{*}} \nabla p_{i}^{(k+1)}\right)+\frac{1}{\Delta t^{2} \rho_{i}^{*}} \frac{\rho_{a 0}}{p_{a 0}} \frac{1}{\gamma} p_{i}^{(k+1)}=-\frac{1}{\Delta t^{2}} \frac{\rho_{a 0}-\rho_{i}^{*}}{\rho_{i}^{*}}+\frac{1}{\Delta t^{2} \rho_{i}^{*}} \frac{\rho_{a 0}}{\gamma}
$$

It is noted that since sound speed is related to gas properties as $\mathrm{c}_{s}=\sqrt{\gamma p / \rho}$, Equation (24) can be converted to the one involving sound speed and can reproduce the propagation of sound (a numerical example will be presented in Section 4.2). However, the advantage of Equation (24) is that it avoids the issue to determine the parameter of actual $c_{s}$ (which is not necessarily constant). This is a significant benefit of the present compressible solver. More importantly, this compressible solver and the previously developed incompressible solver [14] both use the predictor-corrector scheme to solve the same governing equations. The only difference lies in the treatment of fluid density in PPE. The compressible and incompressible solvers can thus be easily integrated leading to the complete twophase model. Named 2P-CPM, it is capable of simultaneously simulating two-phase incompressible and compressible flows with large density difference. The computational procedure is schematically shown in Figure 1 and its performance will be demonstrated in Section 5.

The computational efficiency of 2P-CPM is improved compared to the previous version 2P-CPM which assumes that water and air are both incompressible [14]. The reason is as follows. In the coefficient matrix of the PPE (denoted as $A$ ) generated by applying the derivative-approximation scheme to the first term (incompressible 2P-CPM) of the left hand side of Equation (24), the elements 
in the $i$-th row satisfy $\left|A_{i i}\right|-\sum_{j \neq i}\left|A_{i j}\right|=0\left(A_{i i}>0, A_{i j}<0\right)$ (to meet the diagonal-dominant condition). With the second term in the left hand side of Equation (24), a positive coefficient is added to the diagonal term of the $i$-th row (for air particles). This helps to meet the diagonal-dominant condition and hence makes $A$ matrix better conditioned. As a result, less iteration steps are required to solve the pressure equation and the computational efficiency is improved.

In addition to improved computational efficiency, the better conditioned coefficient matrix of PPE in the 2P-CPM further reduces the spurious pressure fluctuation. Noting that making the diagonal element of the coefficient matrix bigger helps to stabilize the pressure results, Tanaka and Masunaga [37] proposed a quasi-compressibility scheme in MPS by multiplying the diagonal terms with an artificial coefficient which needs to be calibrated. In contrast, the compressible solver in 2P-CPM is derived from gas thermodynamics without any artificial coefficient or artificial sound speed, and is hence more rigorous from physics point of view. Note also that the influence of temperature on air pressure is accounted for in the polytropic gas law.

\section{PERFORMANCE TEST OF NUMERICAL SCHEMES}

\subsection{Accuracy of derivative approximation schemes}

As explained above (more details in [14]), CPM computes spatial derivatives in the framework of Taylor series expansion, achieving better accuracy than the use of kernel function. This advantage is numerically demonstrated by evaluating the Laplacian of a two-dimensional (2D) function $\phi(x, y)=\sin x^{2} \cos y$ with known exact solution. The most commonly used Laplacian operators in MPS [34, 38] and ISPH [33, 39] are adopted here for comparison. Since particles are generally not evenly spaced in a dynamic fluid problem, the Laplacian computation on irregularly spaced particles in the $x-y$ domain of $[3,5] \times[3,5]$ is conducted with initial particle spacing $\left(L_{0}\right) 0.05 \mathrm{~m}$ and influence radius $2.1 L_{0}$. The irregular particles are generated by imposing uniformly distributed random noises within a radius of $0.1 L_{0}$ to the regularly distributed particles $(41 \times 41)$. In Figure 2 , the three numerical solutions of $\nabla^{2} \phi$ are compared with the exact solution. The Laplacian surfaces obtained by MPS and ISPH are not smooth whereas CPM gives a very smooth surface in very good agreement with the exact solution. To test the robustness in accuracy, 100 groups of irregular particle distributions are randomly generated and the global 2-norm errors of the numerical solution of $\nabla^{2} \phi$ are computed. Figure 3 shows that the global errors of MPS and ISPH results are about $13 \%$ and 19\%, respectively, while that of CPM result is only about $2.4 \%$. It shows that, due to the consistent formulation, CPM gives significantly improved accuracy in computing spatial derivatives even for irregular particle distribution. 


\subsection{Pressure wave propagation in an air tube}

Consider the one-dimensional (1D) problem of sound wave in a tube of $25 \mathrm{~m}$ length filled with inviscid air (Figure 4). The air properties at the normal temperature and pressure (NTP) condition are adopted, i.e. density $1.204 \mathrm{~kg} / \mathrm{m}^{3}$ and pressure $1.01325 \times 10^{5} \mathrm{~Pa}$. Pressure wave is generated at the left end of the tube by an oscillating piston governed by $x(t)=s_{\max } \cdot \sin \omega t$, where the oscillating amplitude $S_{\max }$ and frequency $\omega$ are respectively $0.01 \mathrm{~m}$ and $100 \pi \mathrm{rad} / \mathrm{s}$. When the piston is pushed into the tube, a compressed air region, called "compression", is formed. The compression will propagate along the tube and continuously push the region in front of it. Pulling the piston back, however, produces a rarefaction with a lower air density. Theoretically, both the compression and rarefaction propagate in the tube by the sound speed in air, i.e. $c_{s}$ (approximately $343.2 \mathrm{~m} / \mathrm{s}$ in the NTP condition). Based on wave propagation theory, the change in sound pressure can be derived as

$$
\Delta p=\rho_{a} c_{s} \omega s_{\max } \cdot \cos (k x-\omega t)
$$

where $\rho_{a}$ is the air density and $k=\omega / c_{s}$ is the wave number. Accordingly, the amplitude of sound pressure in the air tube is about 1293.1 Pa.

For comparison, 1D numerical simulation by the developed compressible solver is carried out with initial particle displacement $0.02 \mathrm{~m}$ and fixed time step $2.5 \times 10^{-5} \mathrm{~s}$. The wall boundary is modeled by the mirror particle approach [40] in the present paper. Since sound propagation is rapid, it is reasonable to assume an adiabatic condition and adopt $n=1.4$. The predicted pressure distributions in the tube at several time instants are in very good agreement with the analytical solutions as shown in Figure 5. Periodic pressure waves generated at the oscillating source propagate forward. At about 0.07 $\mathrm{s}$, the wave reaches the other end of the tube. Tracking the positions of the wave front, the propagation speed can be determined to be about $338 \mathrm{~m} / \mathrm{s}$, which is very close to the real sound speed in air (with only $1.5 \%$ relative error). Hence, although the sound speed is not a required input, the compressible solver reproduces the sound speed accurately.

\section{VALIDATION FOR 2P-CPM}

The above examples demonstrate the good accuracy of the derivative computation and the developed compressible solver. The capability of 2P-CPM to model water-air flows with entrapped air pocket is validated by three benchmark numerical examples and one experimental study.

\subsection{Water injection into a closed air tube}

The first example of water-air flow involves water injection into a closed air tube as shown in Figure 6a. A gas volume initially contained in a closed tube is compressed when water is injected 
from the bottom end at a constant speed $v$ [41]. According to mass conservation, the analytical solution of air density in the tube is

$$
\rho_{a}(t)=\rho_{a 0} \frac{H_{a 0}}{H_{a 0}-v t}
$$

where $\rho_{a 0}$ is the initial air density at NTP condition and the initial length of air domain is $H_{a 0}=0.5$ $\mathrm{m}$. Since the size of the computational domain is not large, the average pressure in the tube is studied. Assuming the tube to be thermally insulated (i.e. $\gamma=1.4$ ), the air pressure in the tube as a function of time can be obtained by using Equation (18). Numerical simulation is conducted with initial particle spacing $0.005 \mathrm{~m}$ and fixed time step $0.001 \mathrm{~s}$. The water density is taken to be $1000 \mathrm{~kg} / \mathrm{m}^{3}$ and this value will be used in the following water-air flow cases unless otherwise stated.

The snapshot at $t=3.0 \mathrm{~s}$ in Figure $6 \mathrm{~b}$ shows air compression with a clear fluid interface. The relative error of the mean air density is only $1.35 \%$ with respect to the analytical value, i.e. 1.72 $\mathrm{kg} / \mathrm{m}^{3}$ according to Equation (26). For the water domain, its volume is well conserved which can be seen from the good agreement between the simulated water level and the analytical value (the red dash line in Figure 6b). The average air pressure in the tube versus time is presented in Figure 7. The simulated result agrees well with the analytical solution till $3 \mathrm{~s}$ when the compression ratio of air reaches about $45 \%$ (this value is far beyond the compression ratio of air in real wave impact problems). This demonstrates the capability of the 2P-CPM to simulate incompressible and compressible two-phase flows with large density difference (three orders of magnitude) in an integrated way.

\subsection{Oscillating Water Column}

Another validation case of water-air flow is the oscillating water column as shown in Figure $8[4$, 42]. A $1 \mathrm{D}$ closed tube is filled with a water column between two columns of air. Neglecting gravity and viscosity, the water column with an initial velocity $v_{0}$ leads to dynamic motion of the system. Different from the previous example in which the water motion is prescribed, the motion of the water mass is dependent on the response of air columns in this case. Using Equation (18), the air pressure in the tube is

$$
p=p_{a 0}\left(\frac{\rho}{\rho_{a 0}}\right)^{\gamma}=p_{a 0}\left(\frac{H_{a 0}}{H_{a}}\right)^{\gamma}
$$

where $H_{\mathrm{a} 0}$ is the initial length of the air column and $H_{a}$ the length of air column at time $t$. The dynamic equation of water mass can be derived as

$$
. . \quad n \quad \frac{H_{a 0}^{\gamma}}{\rho_{w}}\left(\frac{1}{H_{w}}\left(\frac{1}{\left(H_{a 0}+x\right)^{\gamma}}-\frac{1}{\left(H_{a 0}-x\right)^{\gamma}}\right)\right.
$$


where $x$ and " are respectively the displacement and acceleration of water column, $\rho_{w}$ water density and $H_{w}$ the length of water column. Given the initial boundary conditions, Equation (28) can be solved numerically and used as the benchmark solution.

2P-CPM simulation is conducted with initial particle spacing $0.005 \mathrm{~m}$, fixed time step $0.0001 \mathrm{~s}$ and $\gamma=1.4$. The predicted pressure history at the right end of the tube in Figure 9 shows very good agreement with the analytical solution. As in the previous validation example, the air domains are compressed or expanded while the water volume is well conserved during the 2P-CPM simulation.

\subsection{Large dam break}

The dam break example as shown in Figure 10 contains some important features of practical hydrodynamics problems such as wave slamming on offshore and coastal structures and sloshing impact in containers [18]. In addition, a distinct air pocket is generated, allowing investigation of air cushion effect on impact pressure. In the experimental work by Zhou et al. [43], impact pressure was measured by a pressure sensor located at $160 \mathrm{~mm}$ from the bottom on the right wall. In large scale problems with violent waves, surface tension is normally small compared to others forces; thus surface tension can be neglected in the numerical simulations of this case and the case in the next section. The dynamic viscosities of water and air are $10^{-3} \mathrm{~Pa} \cdot \mathrm{s}$ and $1.983 \times 10^{-5} \mathrm{~Pa} \cdot \mathrm{s}$ respectively in this example (and also in the next example). The initial particle spacing of $0.01 \mathrm{~m}$ (7200 water particles and 30,999 air particles) and fixed time step of $0.0005 \mathrm{~s}$ are used in the 2P-CPM simulation. The computational time is about 0.86 hour per 1000 time steps on a personal computer with i7-2600 CPU (a) $3.40 \mathrm{GHz}$.

The simulated wave profiles at several time instants are shown in Figure 11, in which the right column contains the 2P-CPM results and the left column the 1P-CPM results for comparison. Water flow develops after the dam breaks. At about $0.7 \mathrm{~s}$, water hits and runs up the right wall of the tank. The upward-moving water jet overturns and falls back under gravity to the main water body as a plunging wave which forms an air pocket $(t=1.5 \mathrm{~s})$. The entrapped air pocket deforms in shape $(t=$ 1.5 to $3.0 \mathrm{~s})$ and then breaks near the water surface $(t=3.0 \mathrm{~s})$. The wave profiles predicted by 1PCPM and 2P-CPM are similar for the time period without an entrapped air pocket. It is noted that a clear water-air interface is reproduced by CPM without the use of artificial value for any physical parameter. This is attributed to the novel derivative computation scheme to treat abrupt density and viscosity discontinuity at the water-air interface (details given in [14]). In contrast, some other studies such as Colagrossi and Landrini (2003) have to use an artificial surface tension term to numerically control interface sharpness owing to difficulty in dealing with large density difference across fluid interface. From 1.5 to $3.0 \mathrm{~s}$ with an entrapped air pocket, however, the difference between singlephase and two-phase results is notable. In the 2P-CPM solution, the air pocket exists for some time and its shape keeps changing, because pressure is developed inside the air pocket and offers resistance 
to adjacent water particles (particularly those above the air pocket). In addition, the air pocket vibrates because of air compressibility (the influence of which on impact pressure will be discussed later). In contrast, the water bulk above the entrapment region falls quickly and disappears before $t=2.0 \mathrm{~s}$ in the 1P simulation. This is because the pressure in the entrapment zone is the same as the free-surface boundary condition (zero pressure in the present 1P-CPM simulation) and hence the air pocket offers no resistance (incorrectly) to adjacent water particles.

Figure 12 presents the pressure histories at Point $\mathrm{P}_{1}$ simulated by $1 \mathrm{P}-\mathrm{CPM}$ and $2 \mathrm{P}-\mathrm{CPM}$, the overall trends match well with the experimental result by Zhou et al. [43] (the difference between 1P and $2 \mathrm{P}$ solutions to be discussed later). The first sharp pressure peak, which corresponds to the first impact of water on the right wall (at around $0.7 \mathrm{~s}$ ), has been captured by both the $1 \mathrm{P}$ and $2 \mathrm{P}$ solutions. The slight difference is that the occurrence time of the first peak in the experiment is later than the numerical results. The reason is likely that it was impossible to have a complete dam break instantly in the experiment.

The second pressure peak appears at about $1.5 \mathrm{~s}$ when the overturning flow plunges into the main water body, generating relatively large pressure and creating an air pocket. Since air compressibility is modeled by $2 \mathrm{P}-\mathrm{CPM}$ but not in $1 \mathrm{P}-\mathrm{CPM}$, the fluid pressures near the air pocket predicted by these two methods are different until the disappearance of the air pocket. As shown in Figure 12, the overall trend of water pressure at $\mathrm{P}_{1}$ predicted by $1 \mathrm{P}-\mathrm{CPM}$ gradually decreases as the upward-moving jet falls down. In contrast, the pressure result from 1.5 to $2.3 \mathrm{~s}$ predicted by 2P-CPM exhibits oscillations of relatively large amplitude. It is important to note that this oscillation is physical (not spurious) and is caused by the pressure change in the compressible air pocket (compression and expansion). The pressure in the entrapped air acts on adjacent water particles and hence the water pressure measured at $\mathrm{P}_{1}$ changes with the air-pocket pressure. This explanation can be further supported by Figure 13, in which the average pressure of the air pocket and the fluid pressure at Point $\mathrm{P}_{1}$ fluctuate synchronously in the duration with the existence of air pocket. This shows the importance of capturing the cushion effect of air pocket, which is a significant phenomenon in wave impact scenarios with air entrapment.

It is noted that the experimental result published by Zhou et al. [43] did not show obvious pressure oscillation upon the existence of the air pocket. Since the pressure fluctuation near the first impact peak (see Figure 12) was successfully captured, the pressure sensor used in the experiment should have been able to measure the pressure vibration in the duration with an enclosed air pocket if the pressure vibration did exist. The pressure oscillation might have been filtered out in post processing. Another possible reason is that though the experiment was intended to produce $2 \mathrm{D}$ motion, it was difficult to get a perfectly enclosed air tube. Air could have escaped in the third spatial dimension, thereby diminishing the pressure oscillation. To verify this doubt, a new experiment with targeted measurement of air-pocket pressure and water pressure near the air pocket is designed and conducted in this study (Section 5.4). 
Another remarkable difference between $1 \mathrm{P}$ and $2 \mathrm{P}$ results is that the $1 \mathrm{P}$ simulation predicts a very large pressure peak at around $2.0 \mathrm{~s}$ (see Figure 12) that is not observed in $2 \mathrm{P}$ simulation and was not measured in experiment. This is because the air entrapment region in 1P modeling is effectively vacuum and offers no resistance to water particles around it. Hence water particles above the air entrapment region fall and hit the water body below it, inducing a very large (but unreal) impact pressure. For the same reason, in the single-phase SPH simulations by Colagrossi and Landrini [18] and $\mathrm{Xu}$ [44] (not shown here), this large peak also appears and matches well with 1P-CPM. In contrast, the 2P-CPM solution does not have this large peak and agrees better with the experimental results because the cushion effect in the air entrapment region is modeled accurately. Due to the rebound of the impact water (see the circled cavity region in the 1P-CPM snapshot at $2.08 \mathrm{~s}$ ), another relatively smaller pressure peak is captured by 1P-CPM at about $2.3 \mathrm{~s}$. Thereafter, the 1P-CPM and 2P-CPM results are similar because the influence of the air pocket on fluid motion and pressure becomes very small.

Similar to the 2P-CPM result, the pressure oscillation from 1.5 to $2.3 \mathrm{~s}$ has also been captured in the two-phase SPH simulation as shown in Figure 14. However, the difference between the two-phase $\mathrm{SPH}$ and two-phase CPM results is that the pressure peak (about $5.2 \mathrm{kPa}$ ) at approximately $1.5 \mathrm{~s}$ predicted by $2 \mathrm{P}-\mathrm{CPM}$ agrees better with the experimental result (about $5.0 \mathrm{kPa}$ ) than the pressure peak (about $7.8 \mathrm{kPa}$ ) predicted by $\mathrm{SPH}$.

\subsection{Experimental study with pressure measurement of air pocket}

Benchmark examples of wave impact with entrapped air pockets are limited in the literature, particularly for experimental studies with air pressure measurement. In the dam break case presented in the previous section, although an entrapped air pocket was generated in the experiment, no direct measurement of air pressure change was available for validating numerical results. To fill this gap and further validate the 2P-CPM, sloshing experiments are conducted in a specially designed container so that the shape and pressure variation of an air pocket can be measured. Figure 15 shows the overall experimental setup. The water container (made of Plexiglas plates of thickness $15 \mathrm{~mm}$ ) comprises a big left tank connected by a short channel to a small right tank as shown in Figure 16. This is designed such that, when water in the left tank sloshes to the right (or left), some water will move through the connecting channel and compress (or expand) the air in the right tank. The tank is mounted on a translational shake table (see Figure 15) whose motion is generated by a hydraulic actuator. The input displacement of the shake table is sinusoidal and governed by $x=-A(t) \cdot \sin (\omega t)$, where $A(t)$ and $\omega$ are the amplitude and frequency of the excitation respectively. To avoid a sudden jerk on fluid caused by the non-zero initial velocity of the shake table, the excitation with a linear ramping function $A_{0} t / t_{r}$ is used, where $t_{r}=5 \mathrm{~s}$ is the ramping time and $A_{0}=0.0412 \mathrm{~m}$ is the steady-state amplitude of the excitation. The shake table motion is measured by a displacement transducer and used as input for 
numerical simulation. The air pressure at the middle of the top wall of the right tank, i.e. $\mathrm{P}_{\mathrm{Al}}$ in Figure 16, is measured by an absolute pressure sensor (Model ATM.1ST). Water pressures at $60 \mathrm{~mm}$ from the bottom on the right wall of the right tank $\left(\mathrm{P}_{\mathrm{w}_{1}}\right), 145 \mathrm{~mm}$ from the bottom on the right wall of the left tank $\left(\mathrm{P}_{\mathrm{w}_{2}}\right)$ and $30 \mathrm{~mm}$ from the bottom on the left wall of the left tank $\left(\mathrm{P}_{\mathrm{w}_{3}}\right)$ are measured by gauge pressure sensors (Model WIKA S-10). The wave profiles are recorded by a video camera from the front of the water container. To enhance the clarity of video image, red dye is added to water.

For the designed tank shape, water sloshing with relatively low filling tends to generate larger variation of air pressure in the right tank. This is because the horizontal component of water velocity near the tank bottom is larger in shallow water than in deep water, thereby generating a larger force on water in the connecting channel. But the water level should not be too low so as to maintain a closed air pocket in the right tank. A suitable filling level is $0.17 \mathrm{~m}\left(d_{\mathrm{L}}\right.$ and $d_{\mathrm{R}}$ in Figure 16). Based on some preliminary studies, the excitation frequency of $0.95 \omega_{0}(=3.6807 \mathrm{rad} / \mathrm{s})$ is found to generate a relatively large variation of air pressure in the right tank, where $\omega_{0}$ is the reference frequency computed based on the linear wave theory with water depth $\left(d_{\mathrm{L}}\right)$ and length $\left(L_{\mathrm{L}}\right)$ in the left tank. Therefore, this excitation frequency is used in the following cases.

Based on the experimental work, it is found that there is no entrapped air pocket in the left tank in the cases studied below. In addition, the lid of the left tank (located in the middle of the top wall as shown in Figure 15) is only used to prevent water from gushing out (in cases of very violent sloshing) but not sealed to provide air tightness. Therefore, the air pressure in the void of the left tank is atmospheric pressure during experiments. And hence, air in the void of the left tank is neglected in numerical simulation. The free surface particles are recognized by the "arc" method [11]. In contrast, the right tank is closed (air-tight) and the entrapped air in the void region is included in the simulation as compressible air particles. The fluid properties of water and air are the same as those in the previous case. An initial particle distance of $0.005 \mathrm{~m}$ (7735 water particles and 793 air particles) and fixed time step $0.0005 \mathrm{~s}$ are adopted. The computational time is about 0.57 hour per 1000 time steps by using the computer mentioned in Section 5.3.

The numerical and experimental wave profiles at six time instants are compared in Figure 17, which shows generally good agreement. Because the filling level is low, the water in the left tank moves as a bore. This is consistent with the observations made by Wu et al. [45] and Koh et al. [11]. The bore becomes steeper and its amplitude becomes larger with time (see snapshots at $t=3.56 \mathrm{~s}$ and $4.56 \mathrm{~s}$ in Figure 17). At $6.36 \mathrm{~s}$, the wave approaches the right side of the left tank and generates large impact force on the tank wall and water in the connecting channel (Figure 18c). Consequently, water in the connecting channel is pushed towards right and compresses the enclosed air pocket in the right tank. As expected, a large peak of air pressure appears in the right tank, which is measured in the experiment and predicted well by 2P-CPM (Figure 18a). It is noteworthy that the amplitude of the airpressure change is comparable to the dynamic pressure (i.e. gauge pressure minus the hydrostatic 
pressure) of the very violent and direct wave impact on the tank wall (see Figure 18c). During the impact process from $6.36 \mathrm{~s}$ to $6.96 \mathrm{~s}$, the experimental and numerical results of air pressure in the right tank show vibration and are in good agreement, showing that the pressure vibration in 2P-CPM result is real. The mean air pressure at the wave impact stage is larger than the initial pressure because air is compressed by the impinging water. The air pressure, in turn, influences the water pressure near the air pocket (see water pressure at Point $\mathrm{P}_{\mathrm{w} 1}$ in Figure 18b). The interaction between water and air corroborates the observation in the $2 \mathrm{D}$ dam break case of Section 5.3 that the water pressure at Point $\mathrm{P}_{1}$ on the tank wall oscillates with the pressure in adjacent air pockets.

To demonstrate the spatial convergence of CPM, two cases of particle spacing $\left(l_{0}\right)$ of $0.01 \mathrm{~m}$ and $0.0025 \mathrm{~m}$ are studied in addition to the above-mentioned particle spacing of $0.005 \mathrm{~m}$. As shown in Figure 19, the results of $\mathrm{P}_{\mathrm{A} 1}$ and $\mathrm{P}_{\mathrm{W} 2}$ for $l_{0}=0.005 \mathrm{~m}$ and $l_{0}=0.0025 \mathrm{~m}$ are very similar, whereas the result in the coarsest case $\left(l_{0}=0.01 \mathrm{~m}\right)$ is slightly different from the other two cases. This shows the "grid" convergence of the proposed method. In numerical implementation, the particle spacing is selected to achieve a balance between computational accuracy and efficiency.

For comparison, water sloshing in the same container but without closed air pocket is conducted by drilling holes on the top cover of the right tank to allow air to move in and out freely. Since air gives negligible influence on water motion, this case is simulated as single-phase by 1P-CPM. The same initial particle spacing and time step as those in 2P-CPM simulation are used and the computational time is about 0.3 hour per 1000 time steps on the abovementioned computer. Figure 20 presents the good agreement between the numerical and experimental wave profiles at different time instants. Similar to the sloshing case with enclosed air pocket, the water in the left tank moves as a bore (see $t=3.56 \mathrm{~s}$ and $4.56 \mathrm{~s}$ in Figure 20) and generates large impact forces on tank walls (Figure $21 \mathrm{c}$ and 20d). However, in contrast to the case with enclosed air pocket, the water in the right tank moves up and down violently in the case without enclosed air pocket. When water in the left tank impacts on the right wall, water is pushed into the connecting channel and the water level in the right tank rises up as shown in the snapshots at $6.48 \mathrm{~s}$ and $6.88 \mathrm{~s}$ in Figure 20. Different wave motion further induces different wave pressure at Point $\mathrm{P}_{\mathrm{w} 1}$ as shown in Figure 21 ( $\mathrm{a}$ and $\mathrm{b}$ ). When water moves from the connecting channel to the right tank, flow separation occurs at the top right corner of the connecting channel. This is the reason for the small void in the water domain near the right corner of the connecting channel at $6.48 \mathrm{~s}$. When water in the left tank departs from the connecting channel as shown in the snapshot at $7.56 \mathrm{~s}$, the water level in the right tank drops quickly.

The difference between the wave-impact cases with and without an enclosed air pocket demonstrates the significant influence of air entrapment on wave motion and impact. Therefore, it is essential to conduct two-phase simulation with the consideration of compressible air in many engineering applications involving entrapped air pocket. 
In Section 3.2, it has been explained why the polytropic index $n=1.4$ for air is adopted in the numerical examples of wave impact with entrapped air pocket. To further verify the rationality of this selection, the sloshing case with entrapped air pocket is re-simulated by using $n=1.0$ (isothermal) and $n=1.2$ (an intermediate state between isentropic and isothermal). As shown in Figure 22, the simulated air pressure at $\mathrm{P}_{\mathrm{A} 1}$ using $n=1.4$ (Figure 22a) gives the best agreement with the experimental result. In the other two cases, i.e. $n=1.2$ (Figure 22b) and $n=1.0$ (Figure 22c), the frequencies of air-pressure vibration are smaller than the experimental result. And this discrepancy is more obvious for the case of $n=1.0$. This can be explained by Equation (18) which indicates that, with a smaller polytropic index, a smaller force is required to compress air and hence the stiffness of the simulated air pocket is smaller. Considering the best agreement between the numerical result and the experimental result, the polytropic gas law with $n=1.4$ is recommended for modelling entrapped air pockets in which air compressibility is important and formation of air pockets is relatively fast with little time for heat exchange between the air pocket and the surroundings. This finding is consistent with the analytical studies by Hattori et al. [46] and Abrahamsen and Faltinsen [8].

\section{CONCLUSIONS}

In this study, a new numerical method 2P-CPM is formulated for incompressible and compressible two-phase flows with the following advantageous features.

(1) The first- and second-order derivatives are computed consistently and simultaneously in the framework of Taylor series expansion and do not require kernel function. By using densitynormalized pressure terms in the formulation, abrupt density discontinuity at the fluid interface is handled without the use of smoothing (or smearing) treatment or any other artificial schemes.

(2) A compressible solver is developed for air modelling by employing the polytropic law for ideal gas. The thermodynamic formulation has theoretical rigor and avoids the problem in determining the sound speed (real or artificial). The compressible solver is shown to simulate compression/expansion of air and propagation of pressure wave accurately.

(3) Both the proposed compressible solver and the recently developed incompressible solver for CPM [14] use the same predictor-corrector scheme to solve the governing equations. They are easily integrated to give coupled solution without iteration between the compressible and incompressible solvers.

The capability of 2P-CPM in simulating water-air flows with entrapped air pocket is demonstrated by four numerical examples. For water injection into a closed air tube and oscillating water column, the air pressure and water motion are in good agreement with the analytical solutions and, in particular, a clear fluid interface is reproduced without any special treatment. For the case of large 
dam break, an air pocket trapped by overturning water and its cushion effect characterized by pressure oscillation is successfully simulated by the method.

Finally, an experimental study of water sloshing in a specially designed tank is conducted to measure the pressure change of a closed air pocket under wave impact. Numerical results including wave profiles, sloshing pressures and particularly the pressure vibration in the air pocket predicted by 2P-CPM agree generally well with the experimental results. This shows the accuracy of 2P-CPM and demonstrates the rationality of using polytropic gas law with $\gamma=1.4$ to model air entrapment in wave impact processes. The sloshing case without closed air pocket is also studied experimentally and numerically, showing significant differences from the sloshing case with a closed air pocket. This demonstrates the capability of the proposed 2P-CPM in accounting for compressible air in wave impact problems.

\section{ACKNOWLEDGEMENT}

The authors appreciate the research grant provided by the Singapore Maritime Institute (Project SMI-2014-OF-02) as well as the funding and technical support of Sembcorp Marine Technology Pte Ltd.

\section{REFERENCES}

[1] Liu D, Lin P. A numerical study of three-dimensional liquid sloshing in tanks. Journal of Computational Physics 2008; 227:3921-3939.

[2] Mier-Torrecilla M, Idelsohn SR, Oñate E. Advances in the simulation of multi-fluid flows with the particle finite element method. Application to bubble dynamics. International Journal for Numerical Methods in Fluids 2011; 67:1516-1539.

[3] Wang Y, Shu C, Huang HB, Teo CJ. Multiphase lattice Boltzmann flux solver for incompressible multiphase flows with large density ratio. Journal of Computational Physics 2015; 280:404-423.

[4] Heyns JA, Malan AG, Harms TM, Oxtoby OF. A weakly compressible free-surface flow solver for liquid-gas systems using the volume-of-fluid approach. Journal of Computational Physics 2013; 240:145-157.

[5] Faltinsen OM, Landrini M, Greco M. Slamming in marine applications. Journal of Engineering Mathematics 2004; 48:187-217.

[6] Peregrine DH. Water-wave impact on walls. Annual Review of Fluid Mechanics 2003; 35:23-43.

[7] Peregrine DH, Thais L. The effect of entrained air in violent water wave impacts. Journal of Fluid Mechanics 2006; 325:377- 397. 
[8] Abrahamsen BC, Faltinsen OM. The effect of air leakage and heat exchange on the decay of entrapped air pocket slamming oscillations. Physics of Fluids 2011; 23:102107.

[9] Belytschko T, Lu YY, Gu L. Element-free galerkin methods. International Journal for Numerical Methods in Engineering 1994; 37:229-256.

[10] Idelsohn SR, Oñate E, Pin FD. The particle finite element method: a powerful tool to solve incompressible flows with free-surfaces and breaking waves. International Journal for Numerical Methods in Engineering 2004; 61:964-989.

[11] Koh CG, Gao M, Luo C. A new particle method for simulation of incompressible free surface flow problems. International Journal for Numerical Methods in Engineering 2012; 89:1582-1604.

[12] Liszka T, Orkisz J. The finite difference method at arbitrary irregular grids and its application in applied mechanics. Computers \& Structures 1980; 11:83-95.

[13] Koh CG, Luo M, Gao M, Bai W. Modelling of liquid sloshing with constrained floating baffle. Computers \& Structures 2013; 122:270-279.

[14] Luo M, Koh CG, Gao M, Bai W. A particle method for two-phase flows with large density difference. International Journal for Numerical Methods in Engineering 2015; 103:235-255.

[15] Chorin AJ. A numerical method for solving incompressible viscous flow problems. Journal of Computational Physics 1967; 2:12-26.

[16] Nithiarasu P. An efficient artificial compressibility (AC) scheme based on the characteristic based split (CBS) method for incompressible flows. International Journal for Numerical Methods in Engineering 2003; 56:1815-1845.

[17] Malan AG, Lewis RW, Nithiarasu P. An improved unsteady, unstructured, artificial compressibility, finite volume scheme for viscous incompressible flows: Part I. Theory and implementation. International Journal for Numerical Methods in Engineering 2002; 54:695-714.

[18] Colagrossi A, Landrini M. Numerical simulation of interfacial flows by smoothed particle hydrodynamics. Journal of Computational Physics 2003; 191:448-475.

[19] Yabe T, Wang P-Y. Unified numerical procedure for compressible and incompressible fluid. Journal of the Physical Society of Japan 1991; 60: 2105-2108.

[20] Yoon SY, Yabe T. The unified simulation for incompressible and compressible flow by the predictor-corrector scheme based on the CIP method. Computer Physics Communications 1999; 119:149-158.

[21] Yabe T, Xiao F, Utsumi T. The constrained interpolation profile method for multiphase analysis. Journal of Computational Physics 2001; 169:556-593.

[22] Ikari H, Gotoh H, Sakai T. Simulation of wave run-up by liquid-gas two-phase-flow MPS method. The 3rd International Conference on Asian and Pacific Coasts (APAC 2005), Jeju, Korea, 2005; 1857-1867.

[23] Kieffer SW. Sound speed in liquid-gas mixtures: water-air and water-steam. Journal of Geophysical Research 1977; 82:2895-2904. 
[24] Wemmenhove R. Numerical simulation of two-phase flow in offshore environments. Ph.D. Thesis, University of Groningen, 2008.

[25] Toro EF. Riemann solvers and numerical methods for fluid dynamics: a practical introduction 3rd ed. Heidelberg: Springer, 2009.

[26] Zhang S, Yue DKP, Tanizawa K. Simulation of plunging wave impact on a vertical wall. Journal of Fluid Mechanics 1996; 327:221 - 254.

[27] Hao Y, Prosperetti A. A numerical method for three-dimensional gas-liquid flow computations. Journal of Computational Physics 2004; 196:126-144.

[28] Tofighi N, Yildiz M. Numerical simulation of single droplet dynamics in three-phase flows using ISPH. Computers \& Mathematics with Applications 2013; 66:525-536.

[29] Hu XY, Adams NA. A multi-phase SPH method for macroscopic and mesoscopic flows. Journal of Computational Physics 2006; 213:844-861.

[30] Koshizuka S, Oka Y. Moving-particle semi-implicit method for fragmentation of incompressible fluid. Nuclear science and engineering 1996; 123:421-434.

[31] Belytschko T, Krongauz Y, Organ D, Fleming M, Krysl P. Meshless methods: An overview and recent developments. Computer Methods in Applied Mechanics and Engineering 1996; 139:3-47.

[32] Barrett R, Berry MW, Chan TF, Demmel J, Donato J, Dongarra J, et al. Templates for the Solution of Linear Systems: Building Blocks for Iterative Methods, ed1994.

[33] Shao S, Lo EYM. Incompressible SPH method for simulating Newtonian and non-Newtonian flows with a free surface. Advances in Water Resources 2003; 26:787-800.

[34] Koshizuka S, Nobe A, Oka Y. Numerical analysis of breaking waves using the moving particle semi-implicit method. International Journal for Numerical Methods in Fluids 1998; 26:751-769.

[35] Gotoh H, Sakai T. Key issues in the particle method for computation of wave breaking. Coastal Engineering 2006; 53:171-179.

[36] Fox RW, McDonald AT, Pritchard PJ. Introduction to Fluid Mechanics, 6th ed. New York: Wiley, 2004.

[37] Tanaka M, Masunaga T. Stabilization and smoothing of pressure in MPS method by QuasiCompressibility. Journal of Computational Physics 2010; 229:4279-4290.

[38] Khayyer A, Gotoh H. Modified Moving Particle Semi-implicit methods for the prediction of 2D wave impact pressure. Coastal Engineering 2009; 56:419-440.

[39] Khayyer A, Gotoh H, Shao SD. Corrected incompressible SPH method for accurate watersurface tracking in breaking waves. Coastal Engineering 2008; 55:236-250.

[40] Morris JP, Fox PJ, Zhu Y. Modeling low Reynolds number incompressible flows using SPH. Journal of Computational Physics 1997; 136:214-226.

[41] Caltagirone J-P, Vincent S, Caruyer C. A multiphase compressible model for the simulation of multiphase flows. Computers \& Fluids 2011; 50:24-34. 
[42] Daru V, Le Quéré P, Duluc MC, Le Maître O. A numerical method for the simulation of low Mach number liquid-gas flows. Journal of Computational Physics 2010; 229:8844-8867.

[43] Zhou ZQ, Kat JOD, Buchner B. A nonlinear 3D approach to simulate green water dynamics on deck. Proc 7th Int Conf Num Ship Hydrod, Nantes, France, 1999.

[44] $\mathrm{Xu} \mathrm{H}$. Numercial simulation of breaking wave impact on structures. Ph.D. Thesis, National University of Singapore, 2013.

[45] Wu GX, Ma QW, Eatock Taylor R. Numerical simulation of sloshing waves in a 3D tank based on a finite element method. Applied Ocean Research 1998; 20:337-355.

[46] Hattori M, Arami A, Yui T. Wave impact pressure on vertical walls under breaking waves of various types. Coastal Engineering 1994; 22:79-114.

[47] Kang M, Fedkiw RP, Liu X-D. A boundary condition capturing method for multiphase incompressible flow. Journal of Scientific Computing 2000; 15:323-360.

[48] Sussman M, Smith KM, Hussaini MY, Ohta M, Zhi-Wei R. A sharp interface method for incompressible two-phase flows. Journal of Computational Physics 2007; 221:469-505. 


\section{APPENDIX A}

\section{A.1. Computation of viscosity term for abrupt viscosity discontinuity}

To deal with abrupt viscosity discontinuity, the computation of the viscosity term in Equation (2) is derived by enforcing the continuity of viscous stress (tangential traction) at the two-fluid interface, i.e.

$$
\left(\mu \frac{\partial v}{\partial y}\right)_{i}=\left(\mu \frac{\partial v}{\partial y}\right)_{i+1}=\left(\mu \frac{\partial v}{\partial y}\right)_{I}
$$

where $i$ and $i+1$ are adjacent air and water particles near the interface, and $I$ represents the water-air interface which is the midpoint between particles $i$ and $i+1$ as illustrated in Figure A1.

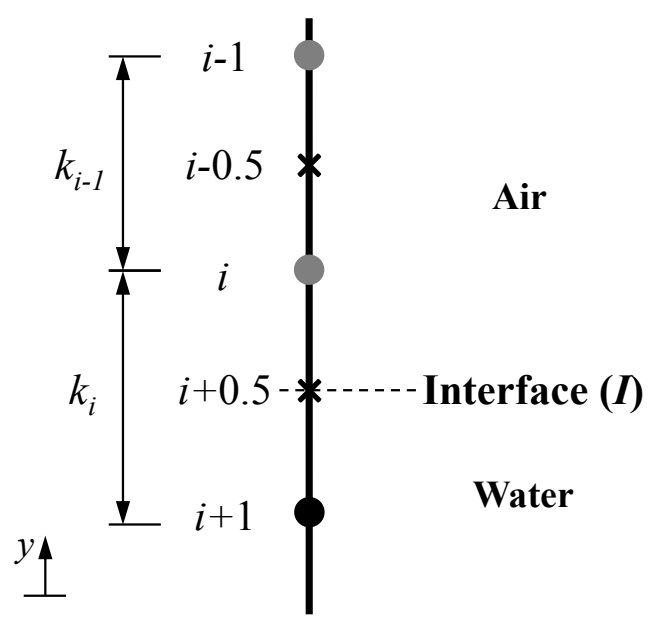

Figure A1. 1-D illustration example of water-air interface

The viscous stress at particle $i$ (air) and particle $i+1$ (water) can be computed by involving particles in their respective fluids as follows

$$
\begin{gathered}
\left(\mu \frac{\partial v}{\partial y}\right)_{i}=\mu_{i} \frac{v_{I}-v_{i}}{0.5 k_{i}} \\
\left(\mu \frac{\partial v}{\partial y}\right)_{i+1}=\mu_{i+1} \frac{v_{i+1}-v_{I}}{0.5 k_{i}}
\end{gathered}
$$

where $v_{i}$ is the velocity at particle $i$ and $v_{I}$ is the velocity at the water-air interface. To enforce continuity of viscous stress at the interface (as the particle spacing approaches zero), we equate the above two equations, resulting in the following interface velocity:

$$
v_{I}=\frac{\mu_{i} v_{i}+\mu_{i+1} v_{i+1}}{\mu_{i}+\mu_{i+1}}
$$

which is the viscosity-weighted average of the velocities at particles $i$ and $i+1$. Substituting Equation (A3) into Equation (A2a) gives 


$$
\left(\mu \frac{\partial v}{\partial y}\right)_{i}=\frac{2}{\mu_{i}^{-1}+\mu_{i+1}^{-1}} \frac{v_{i+1}-v_{i}}{k_{i}}
$$

The above equation implies that the viscosity at interface can be taken as the harmonic mean of viscosities at the particles $i$ (air) and $i+1$ (water) near the interface. Applying finite difference to obtain the spatial derivative of viscosity stress gives

$$
\left(\frac{\partial}{\partial y}\left(\mu \frac{\partial v}{\partial y}\right)\right)_{i}=\left[\left(\mu \frac{\partial v}{\partial y}\right)_{I}-\left(\mu \frac{\partial v}{\partial y}\right)_{i-0.5}\right] \frac{1}{0.5\left(k_{i}+k_{i-1}\right)}
$$

Using Equation (A1) leads to

$$
\left(\mu \frac{\partial v}{\partial y}\right)_{I}=\left(\mu \frac{\partial v}{\partial y}\right)_{i}=\frac{2}{\mu_{i}^{-1}+\mu_{i+1}^{-1}} \frac{v_{i+1}-v_{i}}{k_{i}}
$$

The second term in the right hand side of Equation (A5) can be computed as follows (noting that particles $i$ and $i-1$ are both in the air and hence $\mu_{i-0.5}=\mu_{i}=\mu_{i-1}$ )

$$
\left(\mu \frac{\partial v}{\partial y}\right)_{i-0.5}=\mu_{i-0.5} \frac{v_{i}-v_{i-1}}{k_{i-1}}
$$

which can be, for generality, written as

$$
\left(\mu \frac{\partial v}{\partial y}\right)_{i-0.5}=\frac{2}{\mu_{i-1}^{-1}+\mu_{i}^{-1}} \frac{v_{i}-v_{i-1}}{k_{i-1}}
$$

Substituting Equations (A6) and (A8) into (A5) gives

$$
\left(\frac{\partial}{\partial y}\left(\mu \frac{\partial v}{\partial y}\right)\right)_{i}=\left(\frac{2}{\mu_{i}^{-1}+\mu_{i+1}^{-1}} \frac{v_{i+1}-v_{i}}{k_{i}}-\frac{2}{\mu_{i-1}^{-1}+\mu_{i}^{-1}} \frac{v_{i}-v_{i-1}}{k_{i-1}}\right) \frac{1}{0.5\left(k_{i}+k_{i-1}\right)}
$$

This means that the spatial derivative of viscous stress can be computed by using the harmonic mean of fluid viscosities at the reference and neighbor particles (irrespective of whether the particles are of the same fluid or different fluids). Note that the above equation recovers the special case of singlephase flow by letting $\mu_{i}=\mu_{i+1}=\mu$ :

$$
\left(\frac{\partial}{\partial y} \cdot\left(\mu \frac{\partial v}{\partial y}\right)\right)_{i}=\left(\mu \frac{v_{i+1}-v_{i}}{k_{i}}-\mu \frac{v_{i}-v_{i-1}}{k_{i-1}}\right) \frac{1}{0.5\left(k_{i}+k_{i-1}\right)}
$$

The above formulation is implemented by the GFD scheme which can handle non-uniformly distributed particles, leading to the following derivative computation scheme for the viscosity term with abrupt viscosity discontinuity 


$$
\left(\frac{\partial}{\partial x}\left(\mu \frac{\partial v_{x}}{\partial x}\right)\right)_{i}=\sum_{j \neq i}\left[\frac{2}{\mu_{i}^{-1}+\mu_{j}^{-1}} C_{3 j}\left(v_{x j}-v_{x i}\right)\right]
$$

\section{A.2. Continuity of fluid motion}

The velocity continuity implies that the pressure gradient term $\frac{1}{\rho} \nabla p$ is continuous across fluid interface [47, 48]. In CPM [14], the formulation of density weighted pressure is derived by imposing the following continuity equation at the interface (similarly in the $x$-direction):

$$
\left(\frac{1}{\rho} \frac{\partial p}{\partial y}\right)_{i}=\left(\frac{1}{\rho} \frac{\partial p}{\partial y}\right)_{i+1}=\left(\frac{1}{\rho} \frac{\partial p}{\partial y}\right)_{I}
$$

where $i$ and $i+1$ are adjacent air and water particles near the fluid interface $I$ (Figure A1). Hence, the continuity condition of fluid motion is achieved at the interface (as the particle spacing approaches zero).

\section{A.3. Continuity of traction force}

The traction continuity condition requires the tangential fluid stress due to viscous force be continuous. As shown in Section A.1, the viscosity-weighted velocity is in fact derived by enforcing continuity of viscous stress at the two-fluid interface. Thus, the continuity condition of tangential traction is ensured.

For normal traction, the continuity condition requires that the water and air pressures be the same at the interface (if surface tension force is neglected), i.e.

$$
p_{I}^{W}=p_{I}^{A}
$$

where $p_{I}^{W}$ and $p_{I}^{A}$ are the water and air pressure at the fluid interface respectively. In CPM [14], the pressure gradients at particles $i$ (air) and $i+1$ (water) near the interface are computed as follows

$$
\left(\frac{1}{\rho} \frac{\partial p}{\partial y}\right)_{i}=\frac{1}{\rho_{i}} \frac{p_{I}^{A}-p_{i}}{0.5 k_{i}}
$$

and

$$
\left(\frac{1}{\rho} \frac{\partial p}{\partial y}\right)_{i+1}=\frac{1}{\rho_{i+1}} \frac{p_{i+1}-p_{I}^{W}}{0.5 k_{i}}
$$

The continuity of normal traction is enforced by setting $p_{I}^{W}=p_{I}^{A}=p_{I}$ in CPM. 


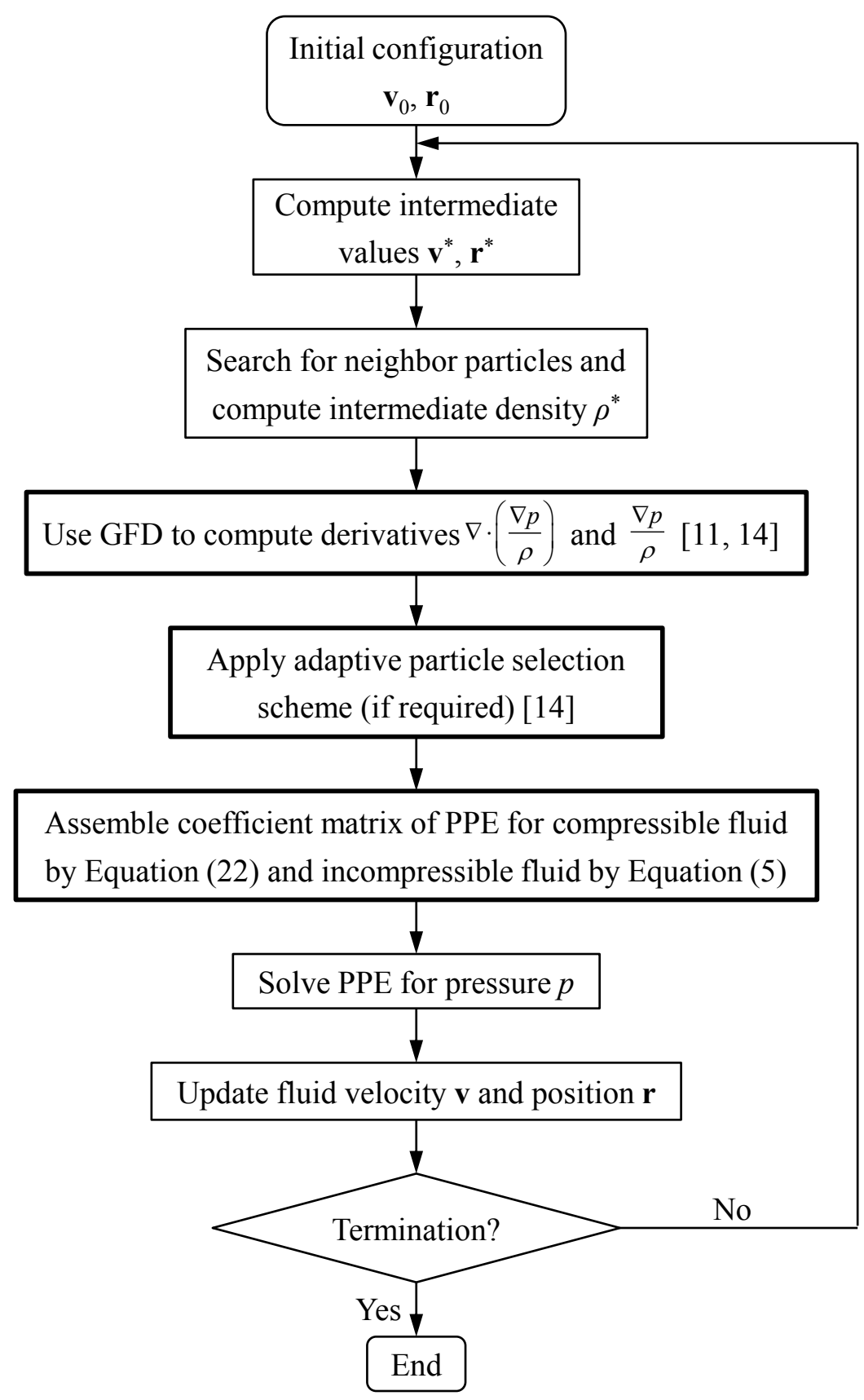

Figure 1. Flow chart of 2P-CPM for two-phase incompressible and compressible flows with large density difference 

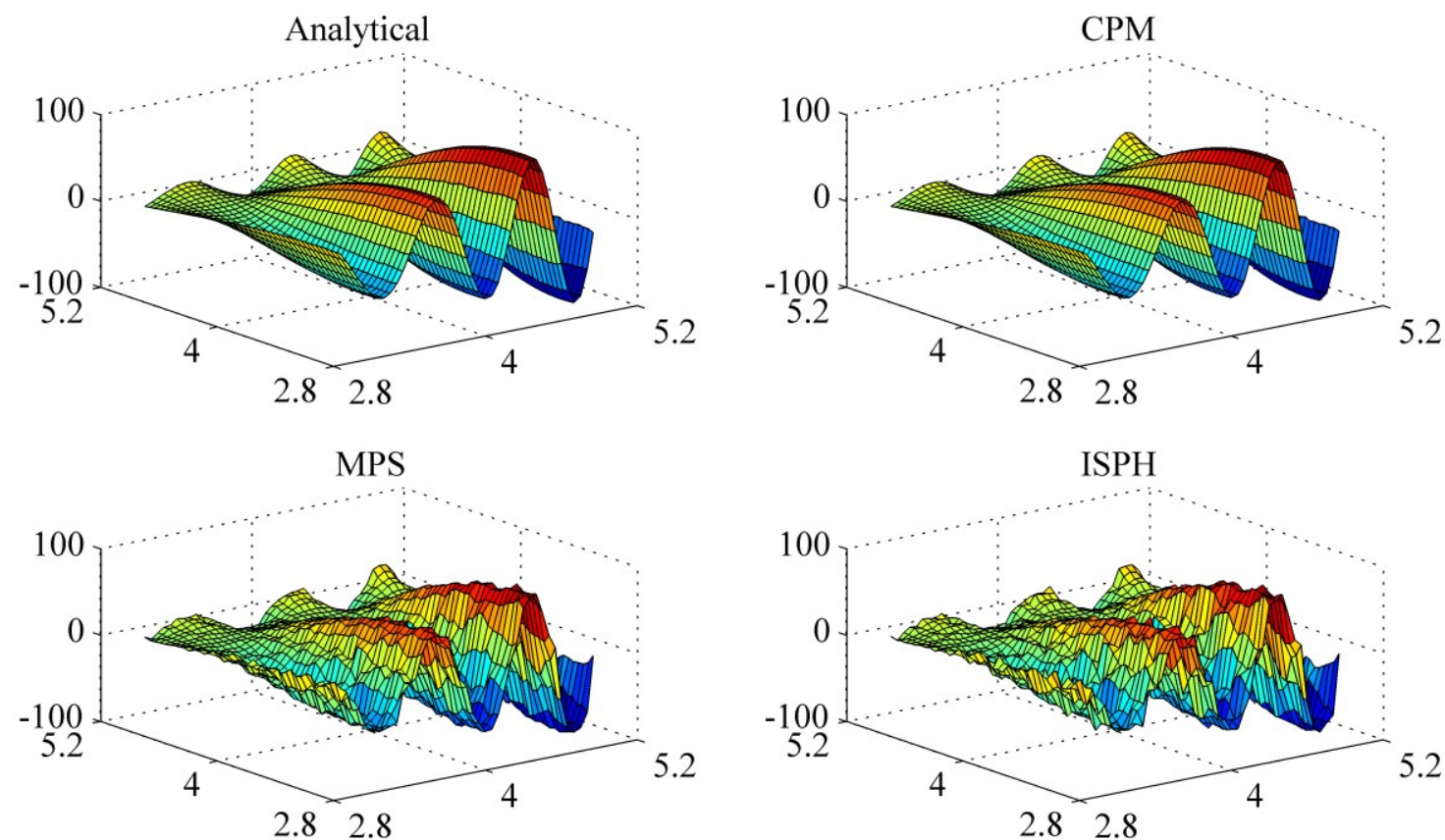

Figure 2. Analytical and numerical results of the Laplace of a 2D function

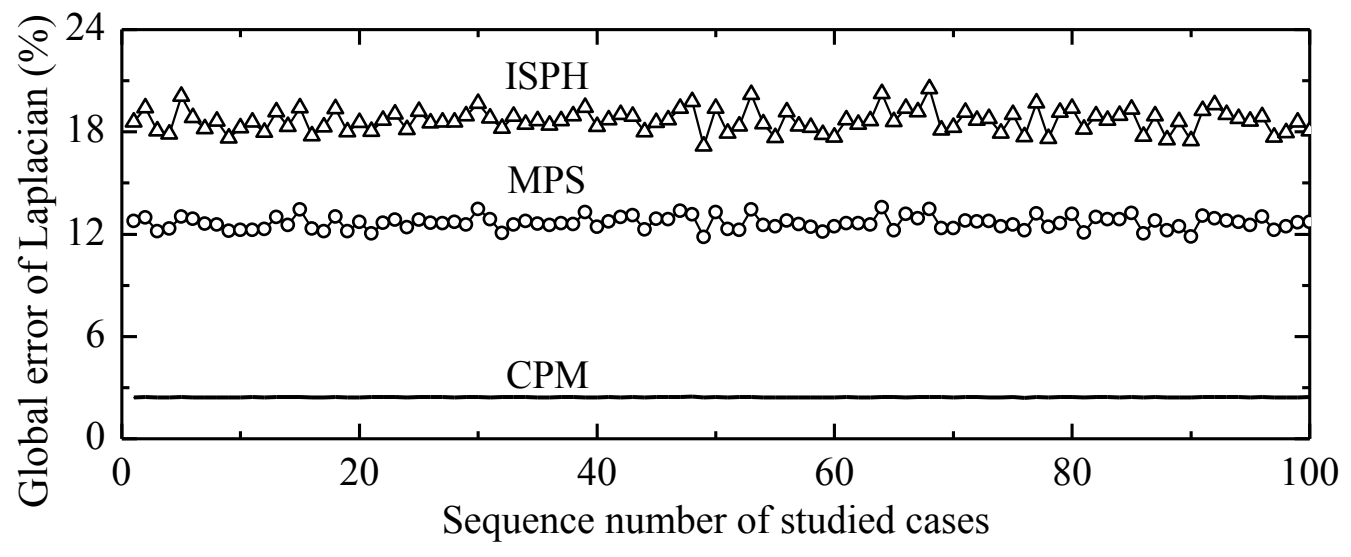

Figure 3. Global error of Laplacian approximation by CPM, MPS and ISPH 


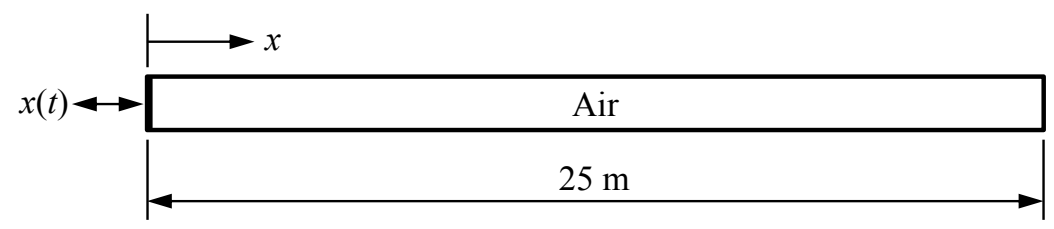

Figure 4. Schematic view of pressure wave propagation in an air tube

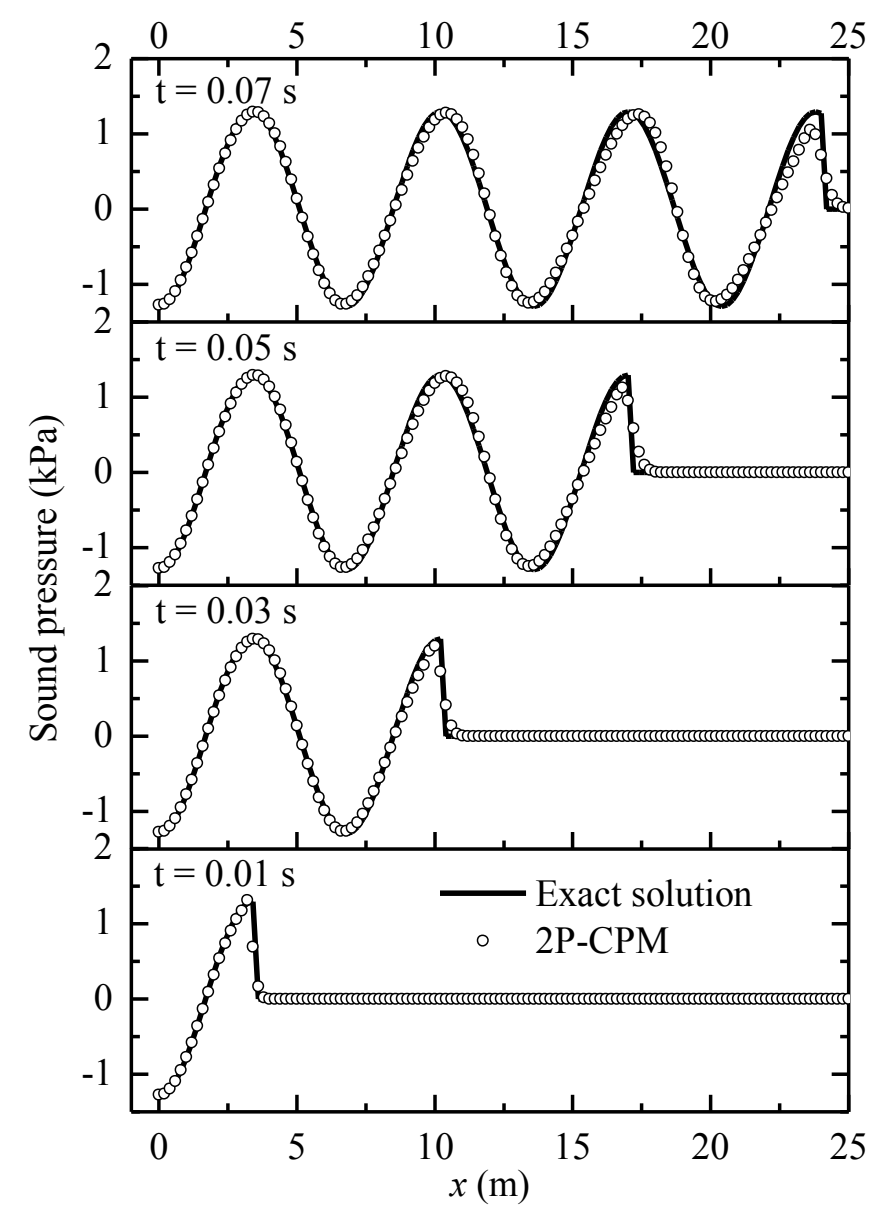

Figure 5. Sound pressure distributions along the tube at several time instants 


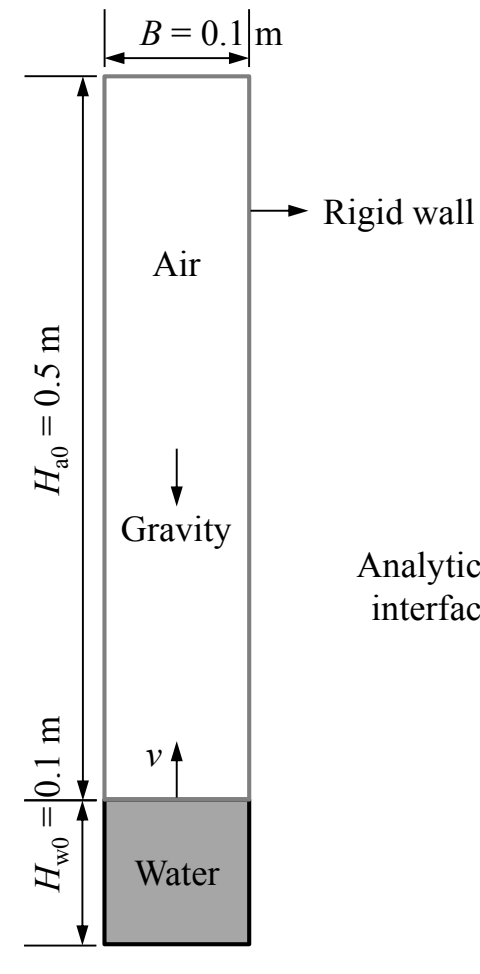

(a)

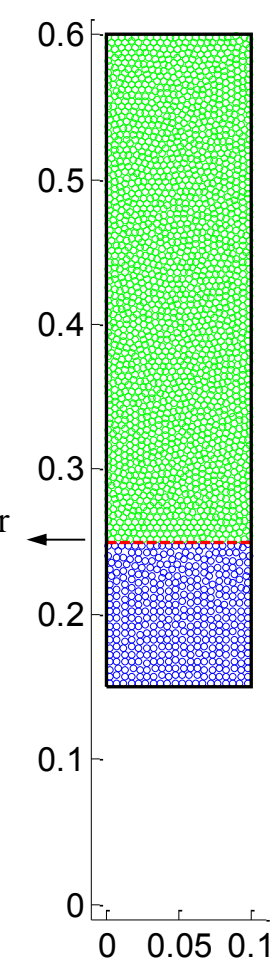

(b)

Figure 6. Water injection into a closed air tube: (a) initial configuration, (b) snapshot at $t=3.0 \mathrm{~s}$

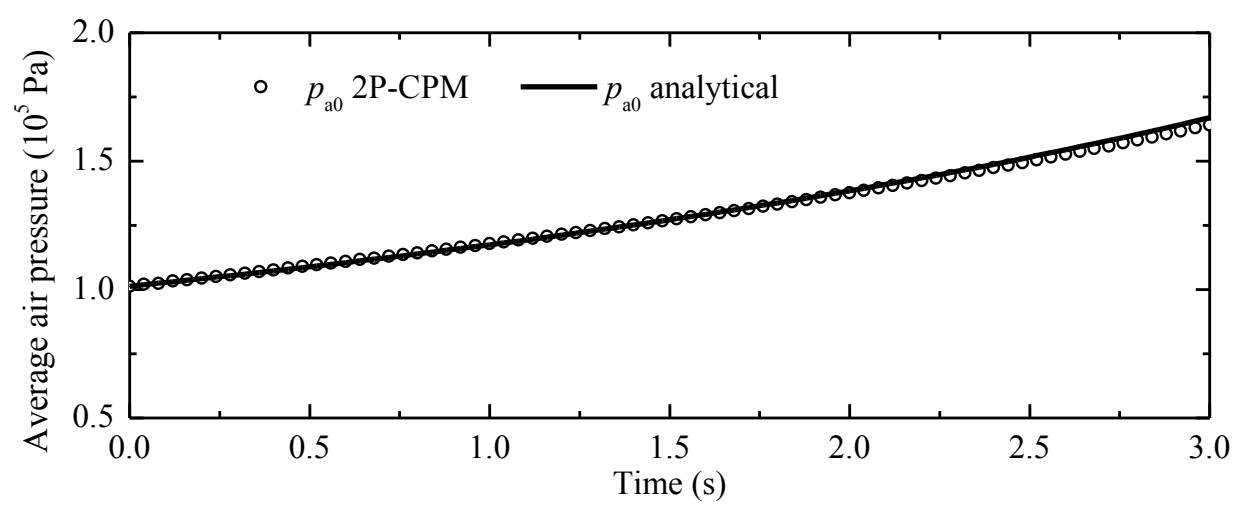

Figure 7. Water injection into a closed air tube: average air pressure

\begin{tabular}{|c|c|c|c|}
\hline Air & Water & $\longrightarrow v_{0}$ & Air \\
\hline$H_{\mathrm{a} 0}=0.9 \mathrm{~m}$ & $H_{\mathrm{w}}=$ & $0.2 \mathrm{~m}$ & $H_{\mathrm{a} 0}=0.9 \mathrm{~m}$ \\
\hline
\end{tabular}

Figure 8. Oscillating water column: initial configuration 


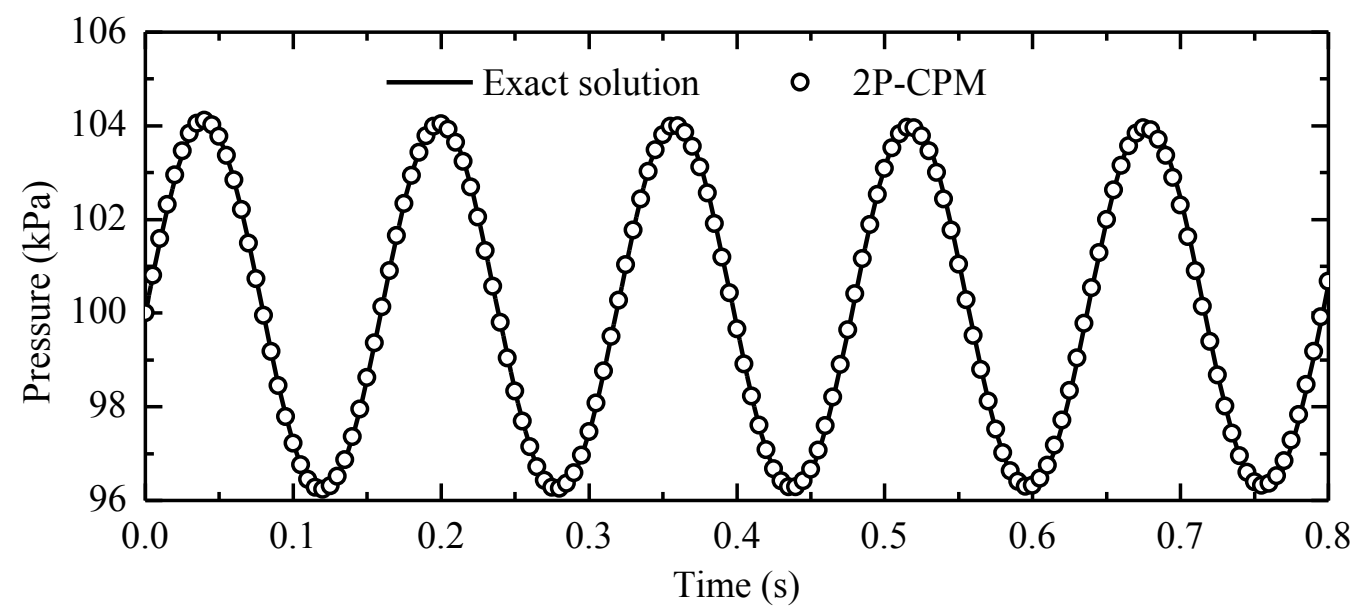

Figure 9. Oscillating water column: pressure history on the right wall of the tube

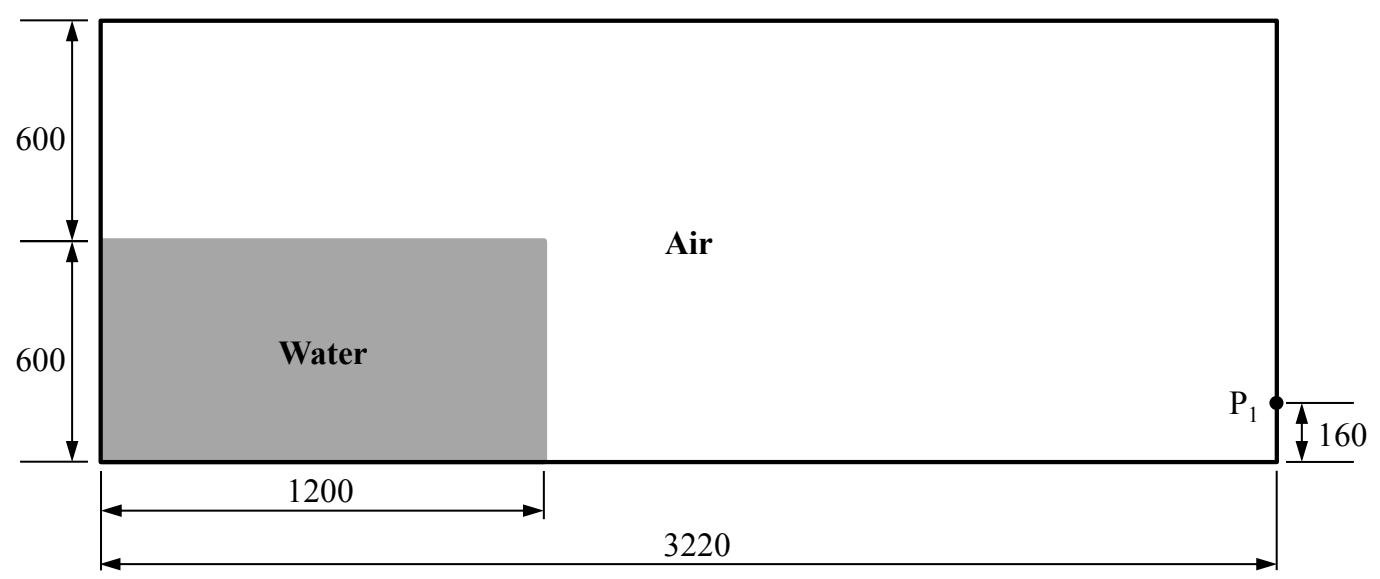

Figure 10. Large dam break: initial configuration (Unit: $\mathrm{mm}$ ) 


\section{P-CPM}

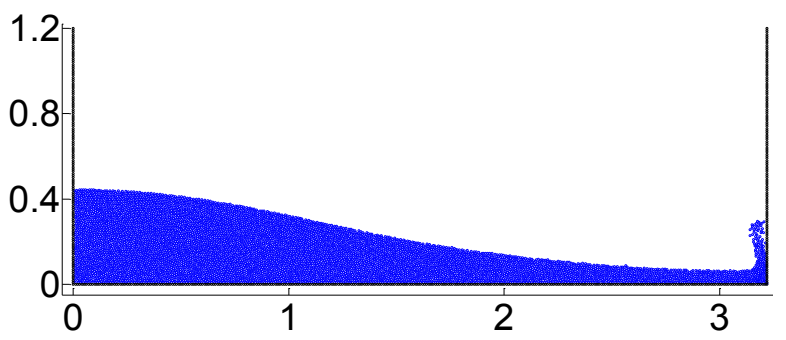

2P-CPM

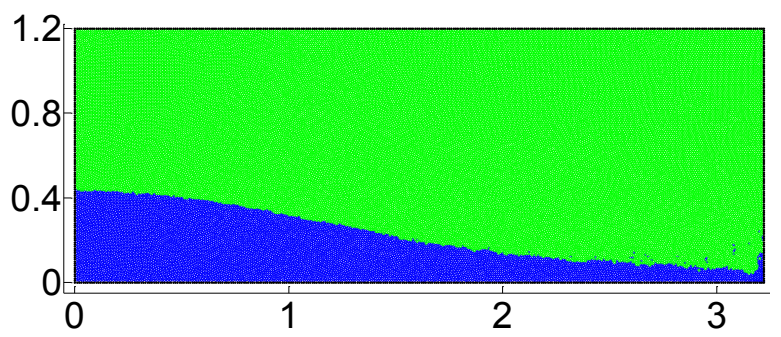

$t=0.7 \mathrm{~s}$
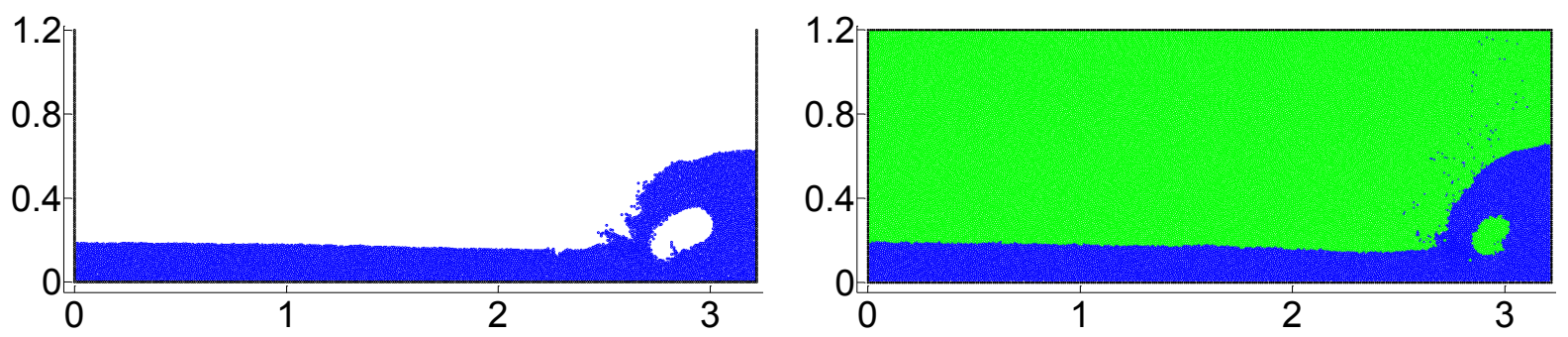

$$
t=1.5 \mathrm{~s}
$$
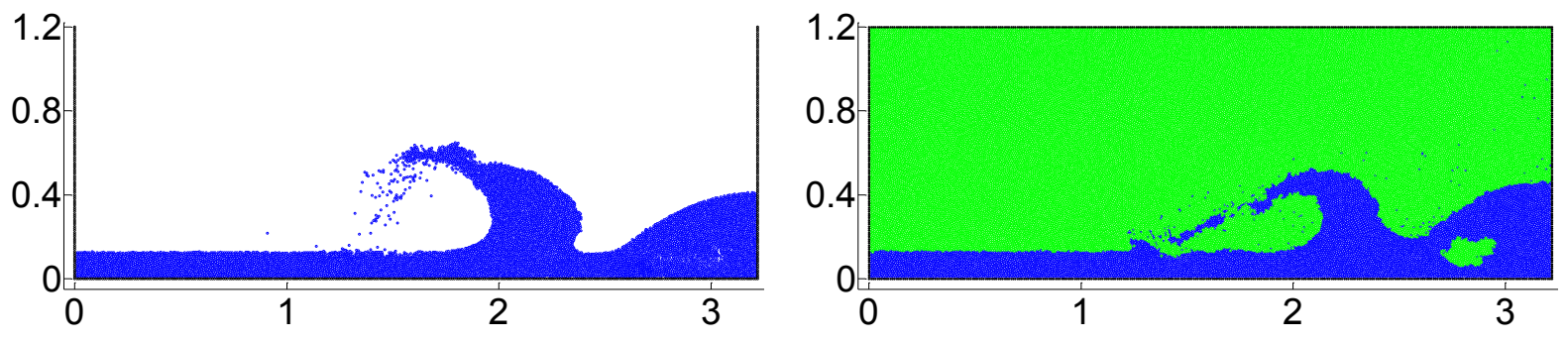

$$
t=2.0 \mathrm{~s}
$$
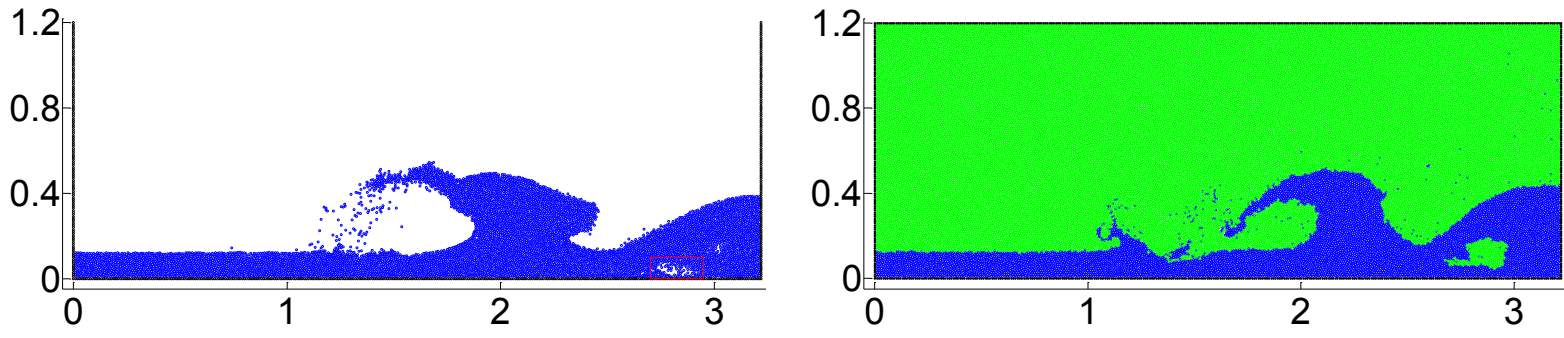

$$
t=2.08 \mathrm{~s}
$$
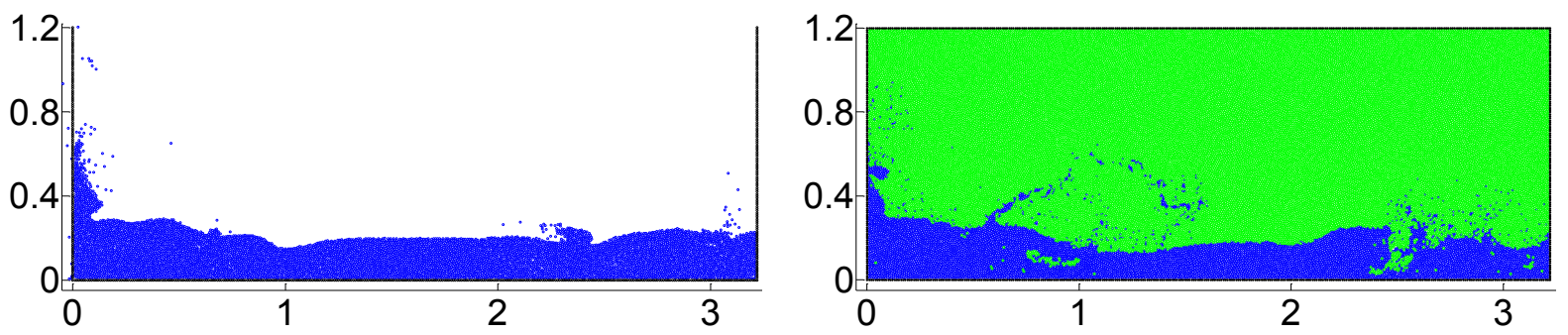

$t=3.0 \mathrm{~s}$

Figure 11. Large dam break: predicted wave profiles by 1P-CPM and 2P-CPM 


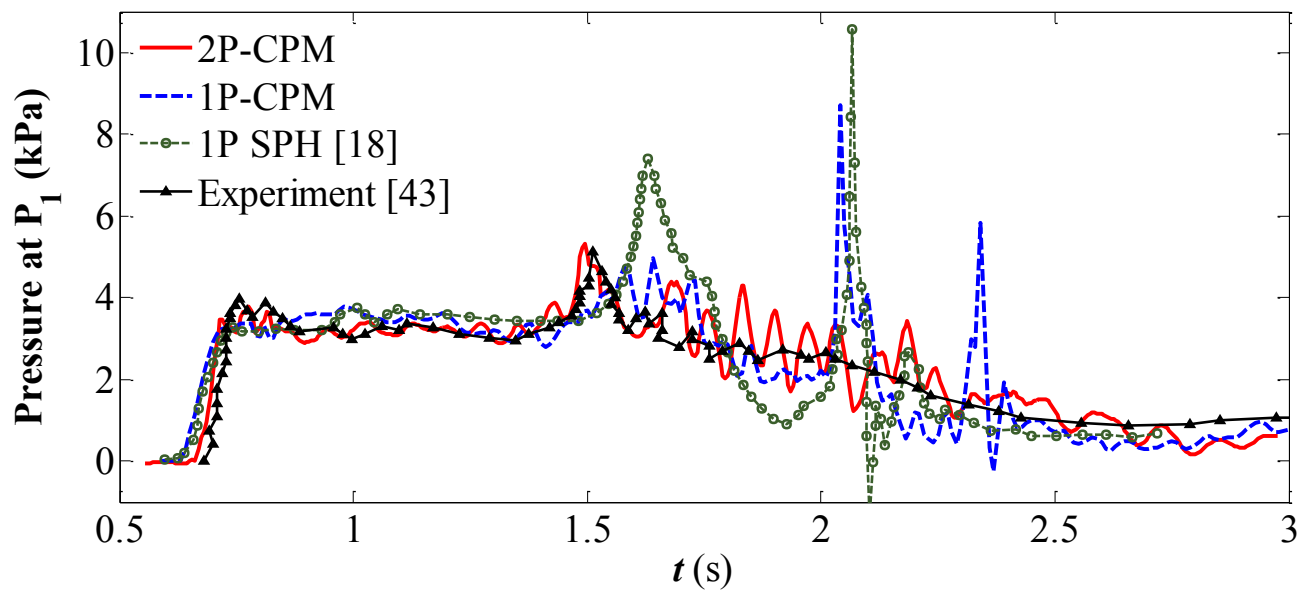

Figure 12. Large dam break: predicted pressure history at Point $\mathrm{P}_{1}$ by 1P-CPM and 2P-CPM in comparison with experimental result by Zhou et al. [43] and 1P SPH result by Colagrossi and Landrini [18]

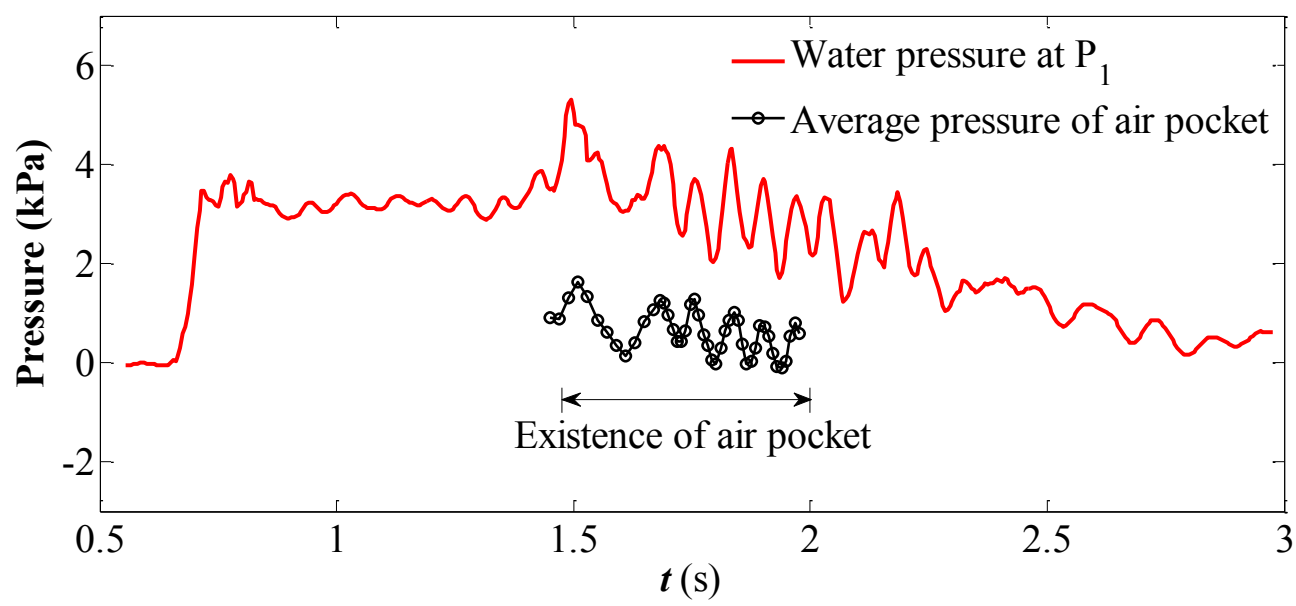

Figure 13. Large dam break: water pressure at Point $P_{1}$ and average pressure of air pocket predicted by $2 \mathrm{P}-\mathrm{CPM}$

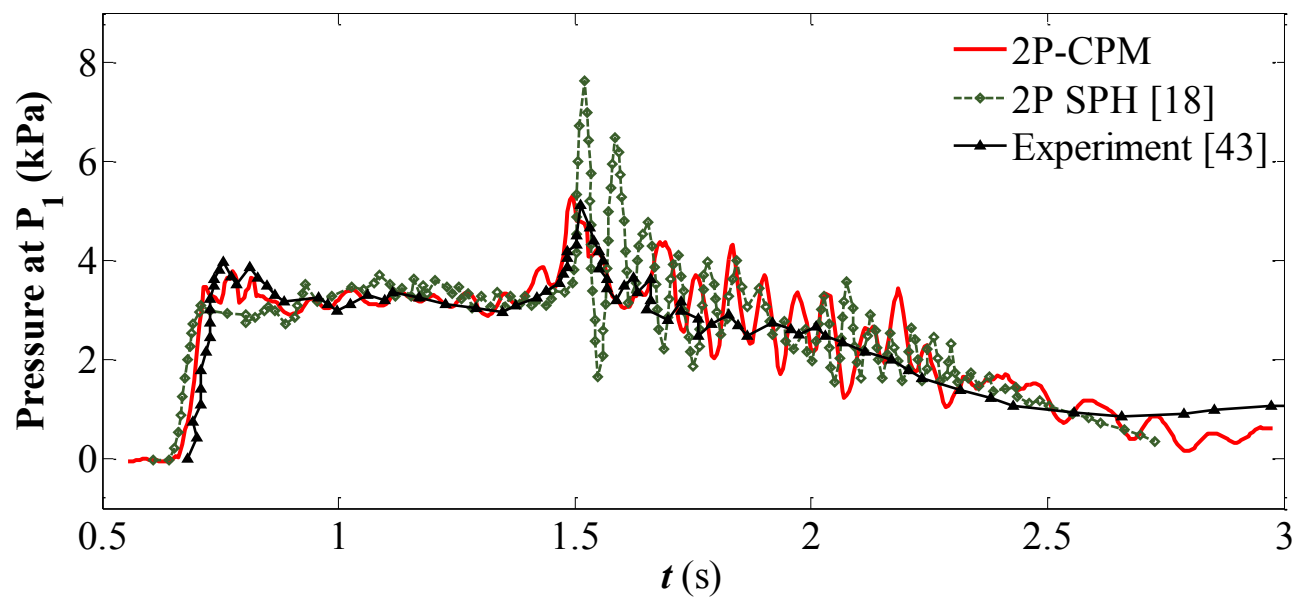

Figure 14. Large dam break: predicted pressure history at Point $\mathrm{P}_{1}$ by $2 \mathrm{P}-\mathrm{CPM}$ in compassion with experimental result by Zhou et al. [43] and 2P SPH result by Colagrossi and Landrini [18] 


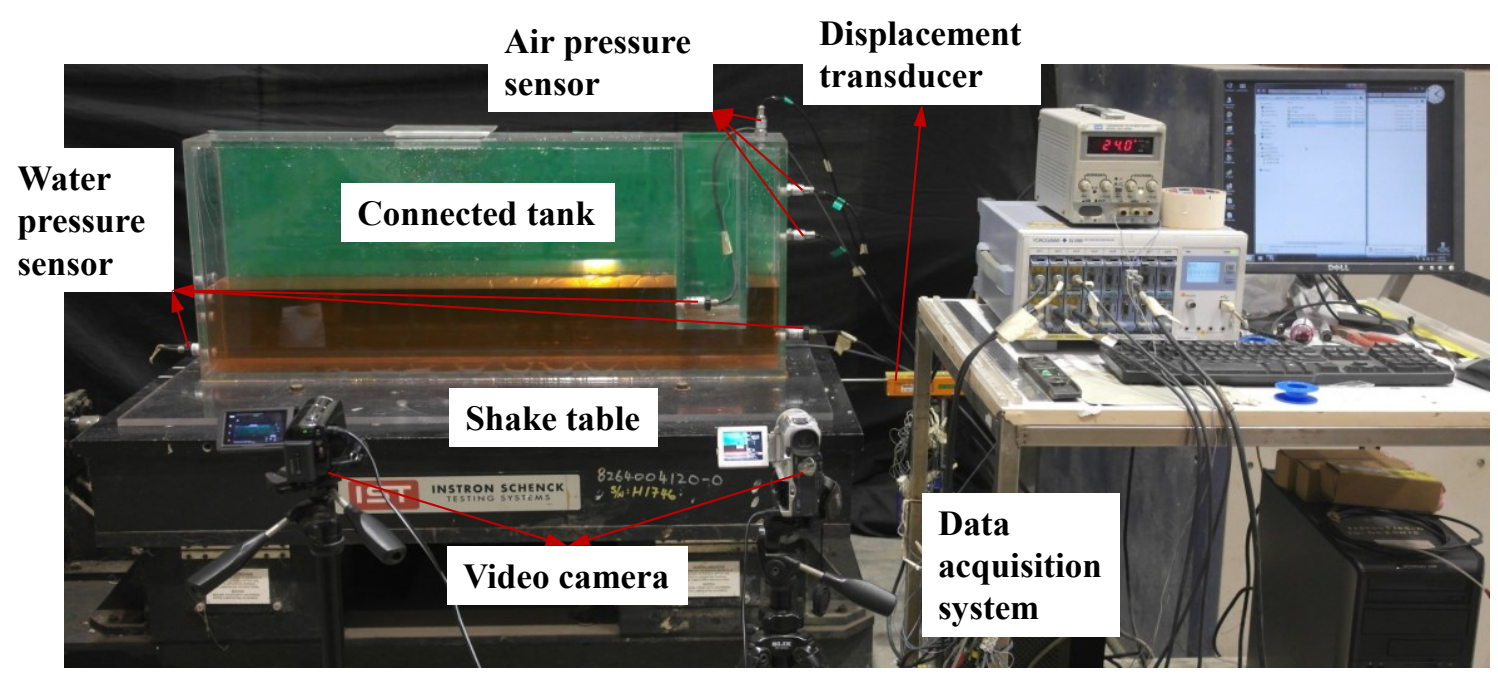

Figure 15. Water-air sloshing in a connected container under translational excitation

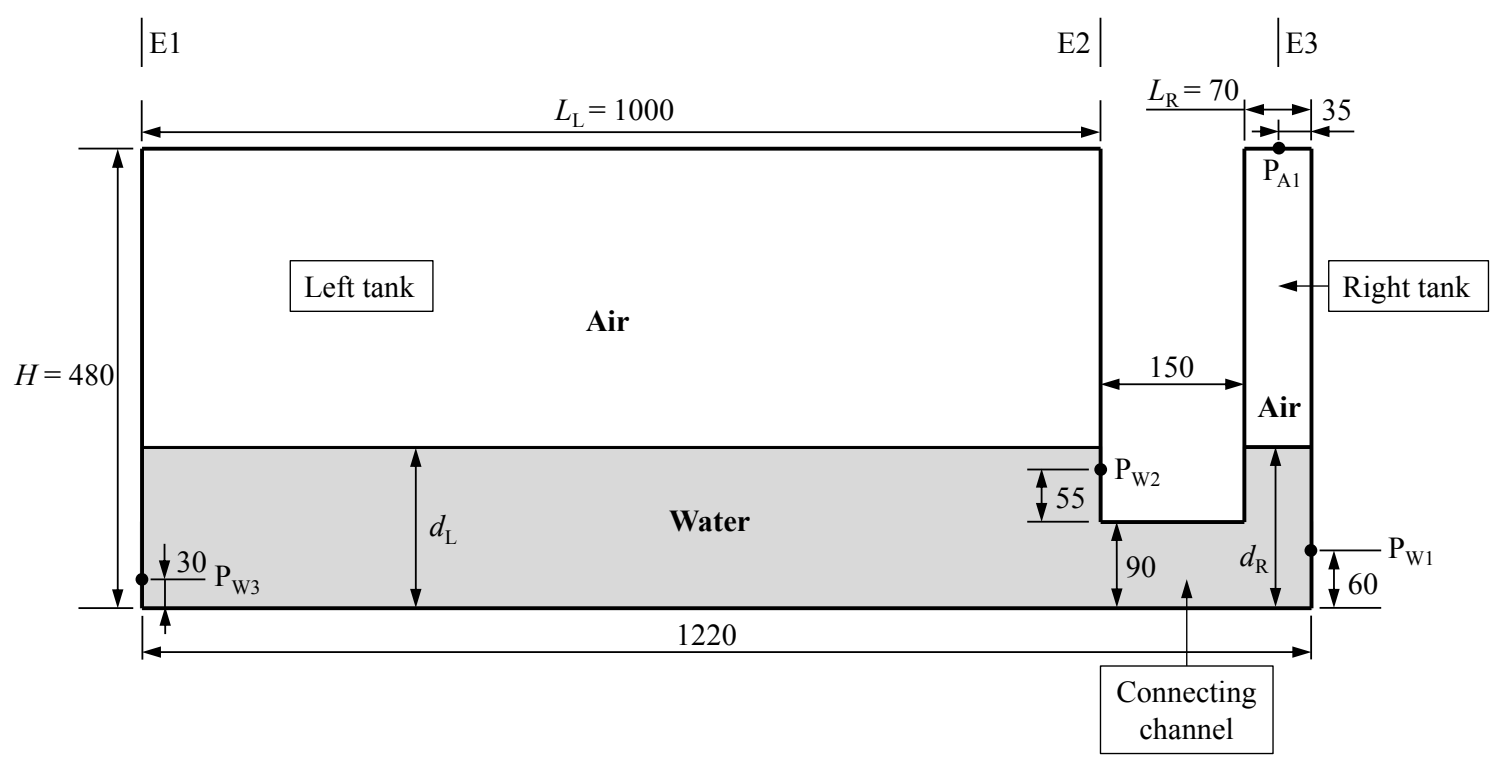

Figure 16. Geometric dimensions of the connected container used in sloshing experiments (Unit: $\mathrm{mm}$ ) 
Experiment
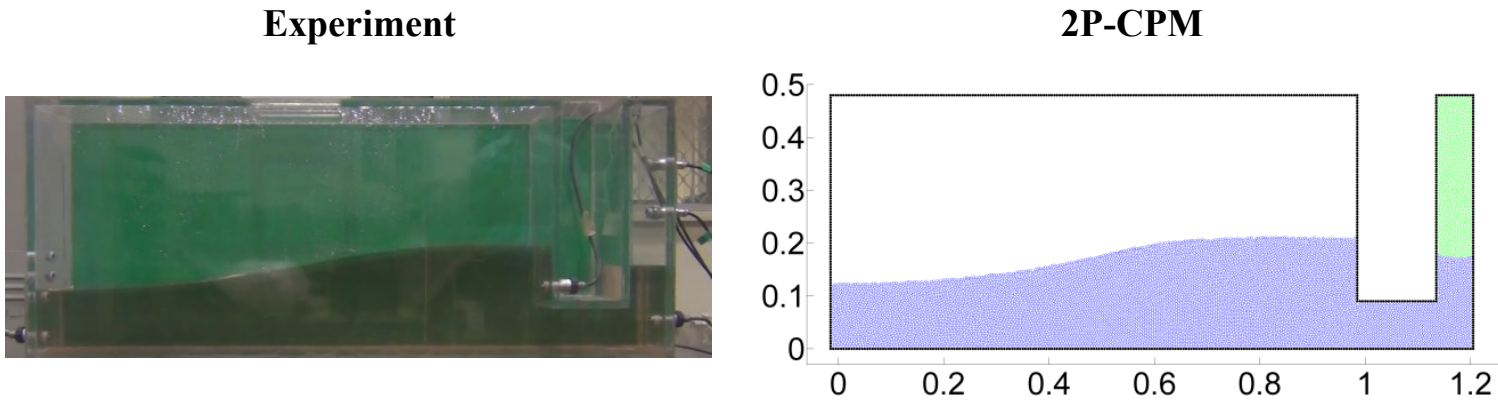

$t=3.56 \mathrm{~s}$
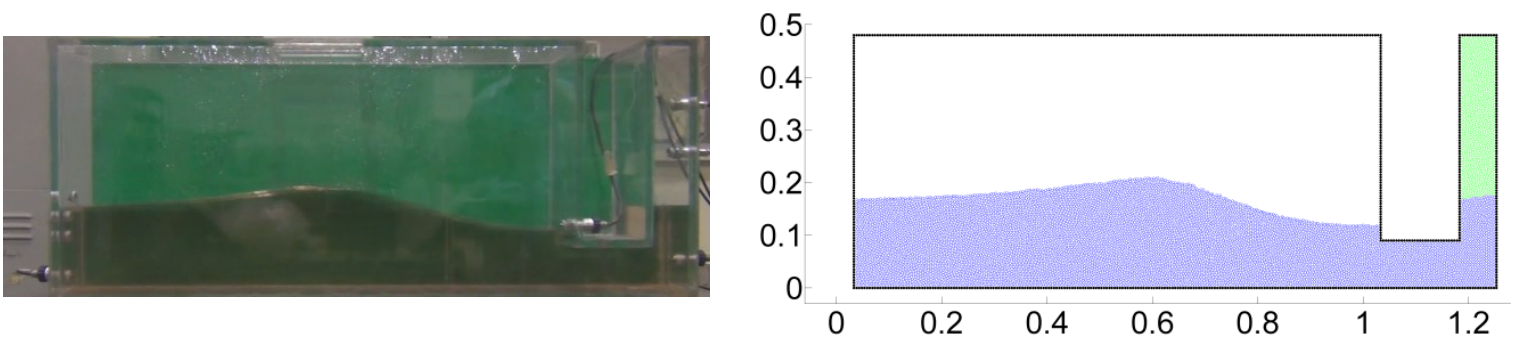

$t=4.56 \mathrm{~s}$
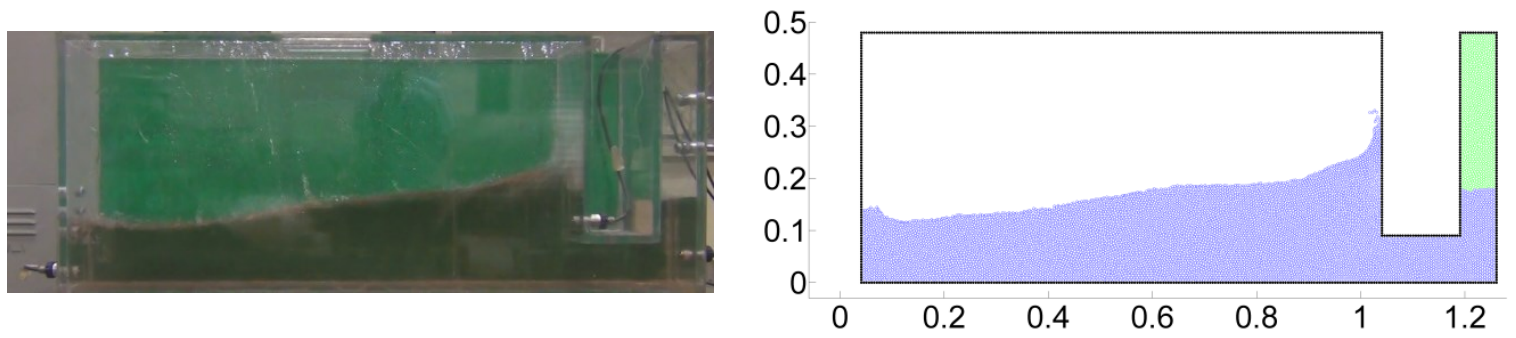

$$
t=6.36 \mathrm{~s}
$$
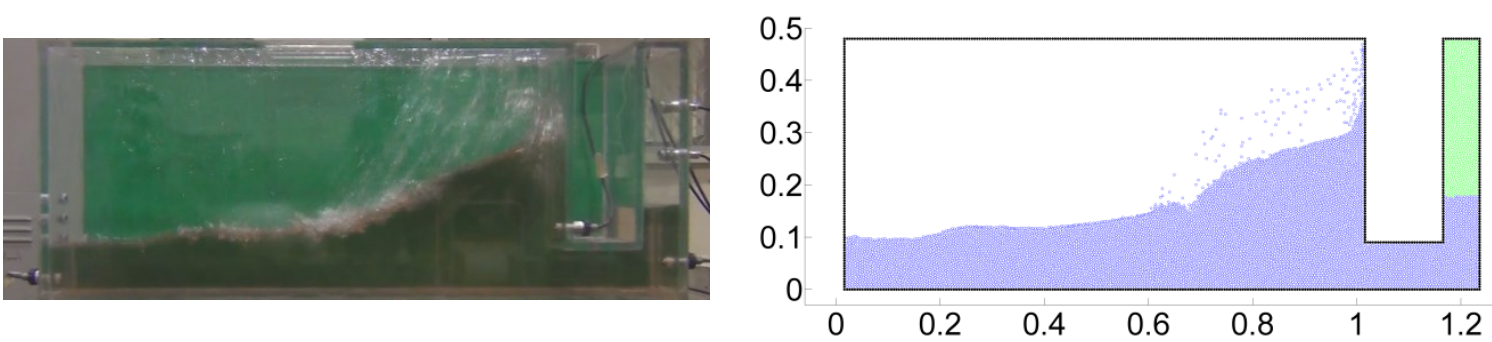

$$
t=6.72 \mathrm{~s}
$$
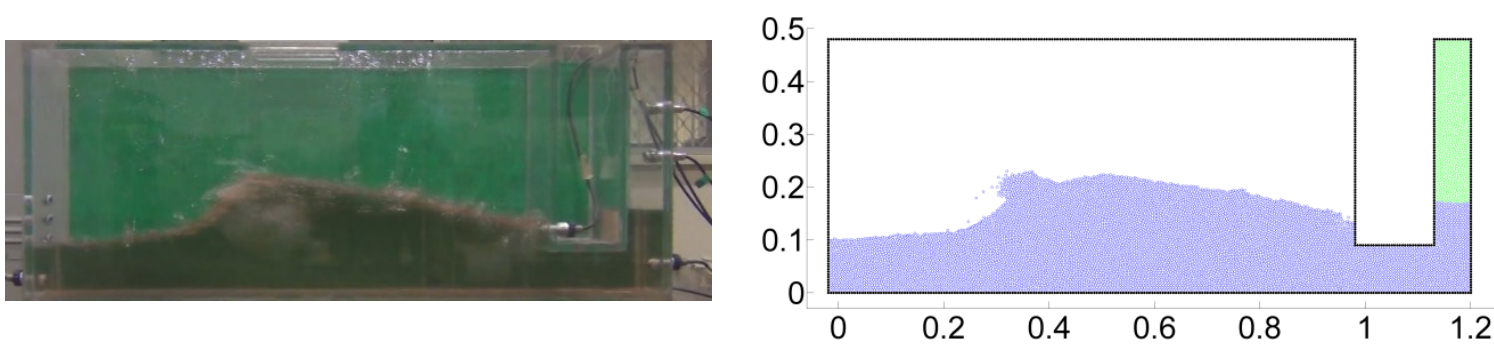

$$
t=6.96 \mathrm{~s}
$$

Figure 17. Wave profiles of sloshing with closed air pocket: experimental result and 2P-CPM simulation 


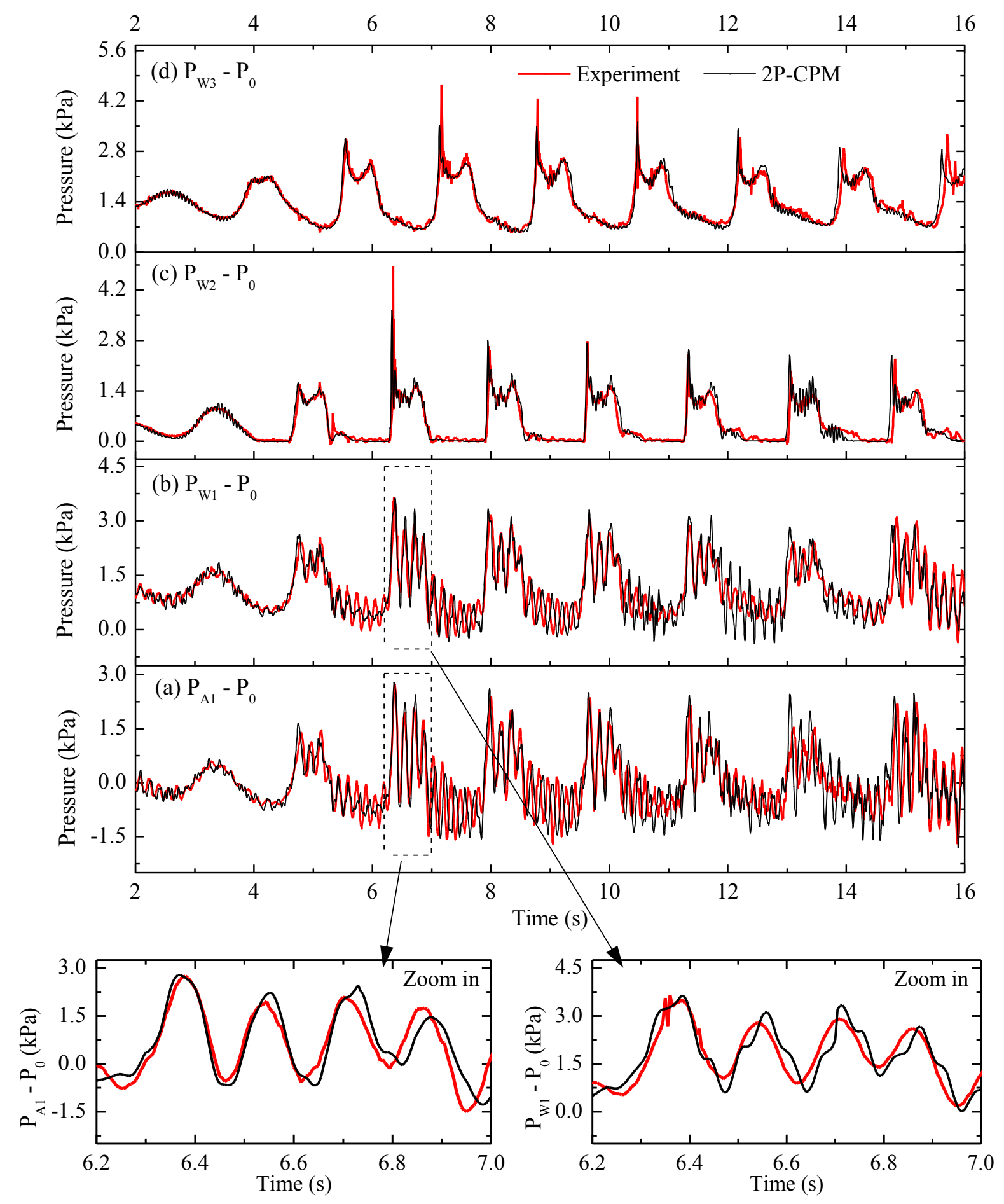

Figure 18. 2P-CPM results of air pressure at Point $\mathrm{P}_{\mathrm{A} 1}$ and water pressures at Point $\mathrm{P}_{\mathrm{W} 1}, \mathrm{P}_{\mathrm{W} 2}$ and $\mathrm{P}_{\mathrm{W} 3}$ in comparison with experimental results (with enclosed air pocket) 

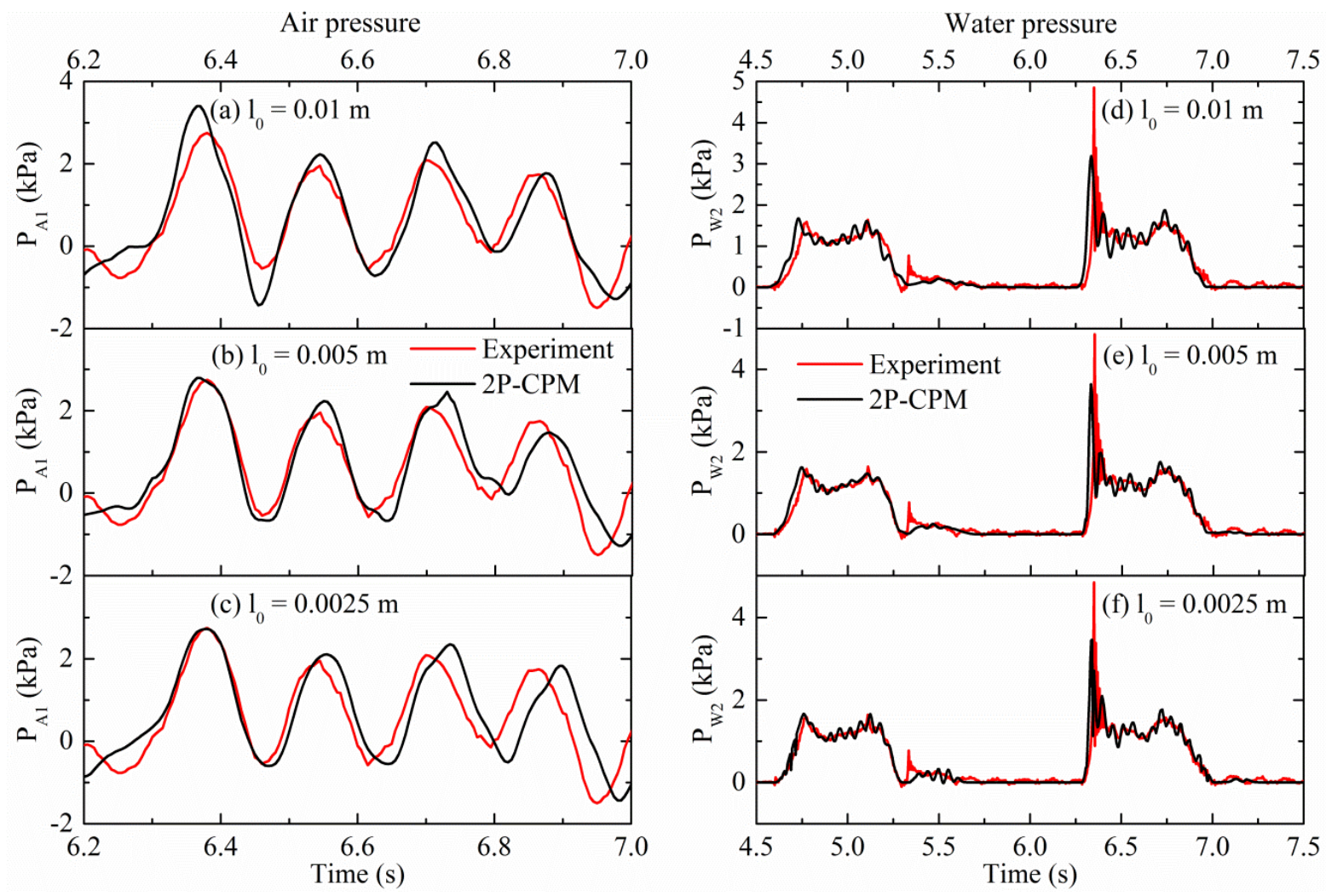

Figure 19. Comparison of $\mathrm{P}_{\mathrm{A} 1}$ and $\mathrm{P}_{\mathrm{W} 2}$ at initial particle spacing of $0.01 \mathrm{~m}, 0.005 \mathrm{~m}$ and $0.0025 \mathrm{~m}$ 
Experiment
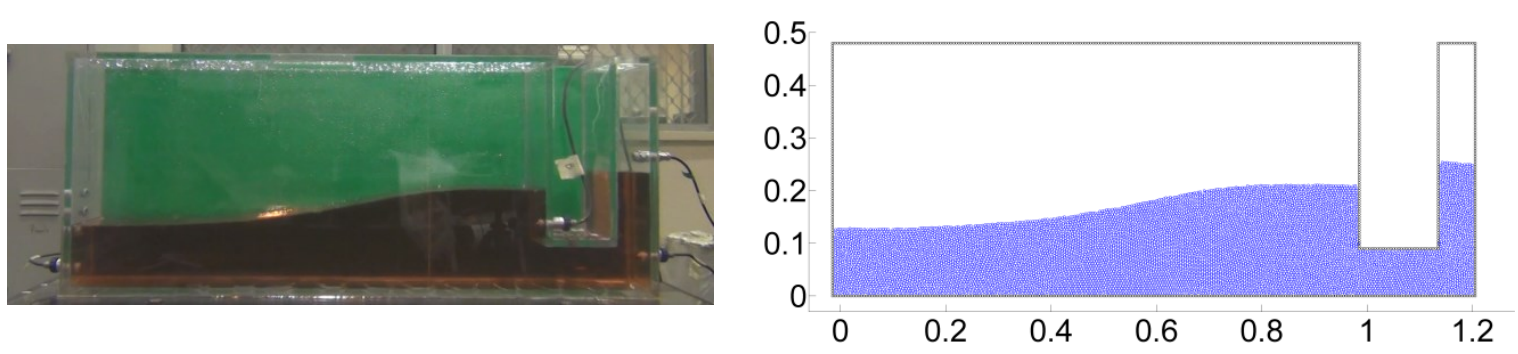

$t=3.56 \mathrm{~s}$
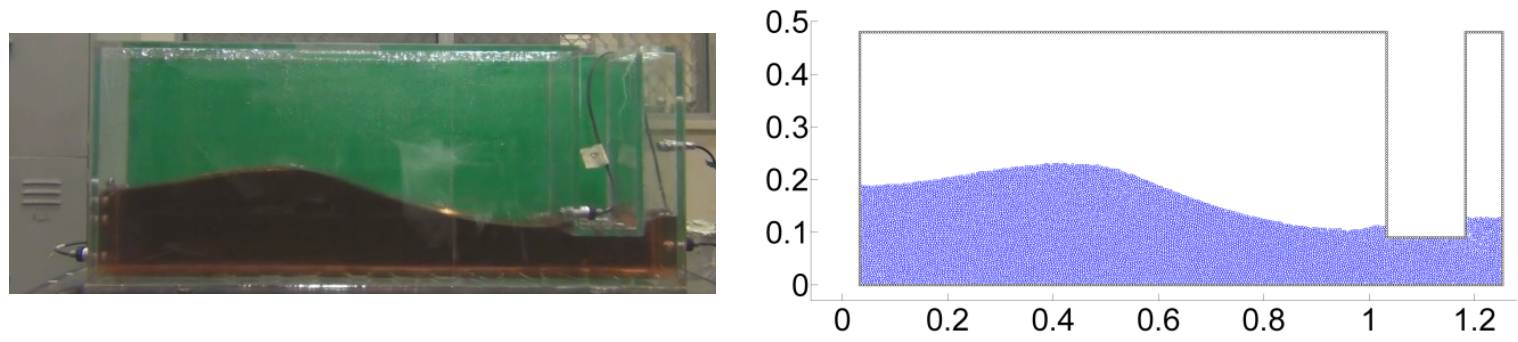

$t=4.56 \mathrm{~s}$
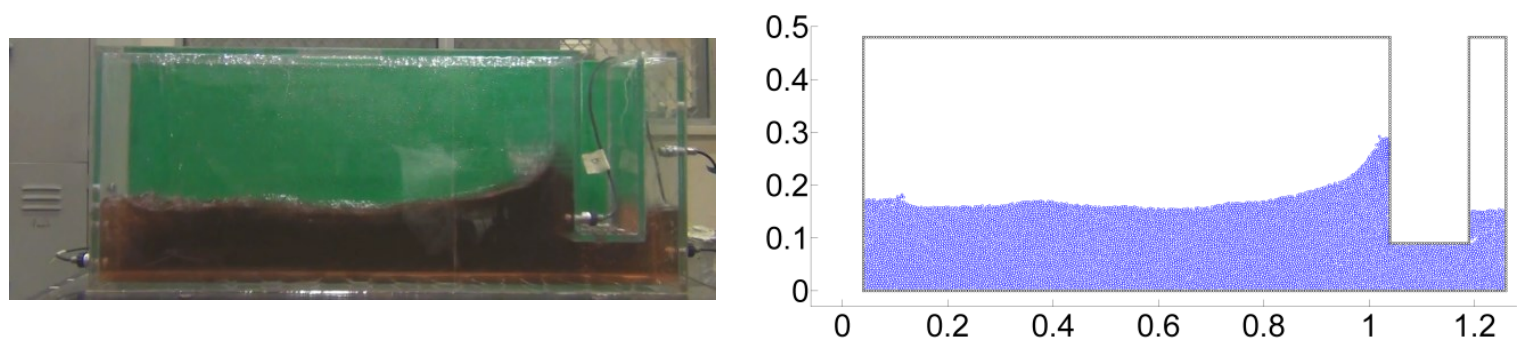

$$
t=6.48 \mathrm{~s}
$$
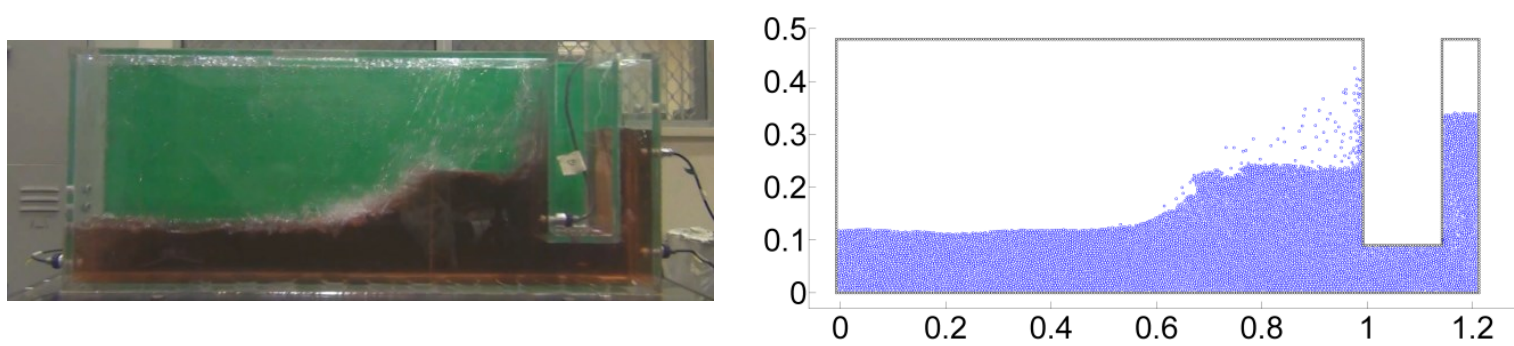

$$
t=6.88 \mathrm{~s}
$$
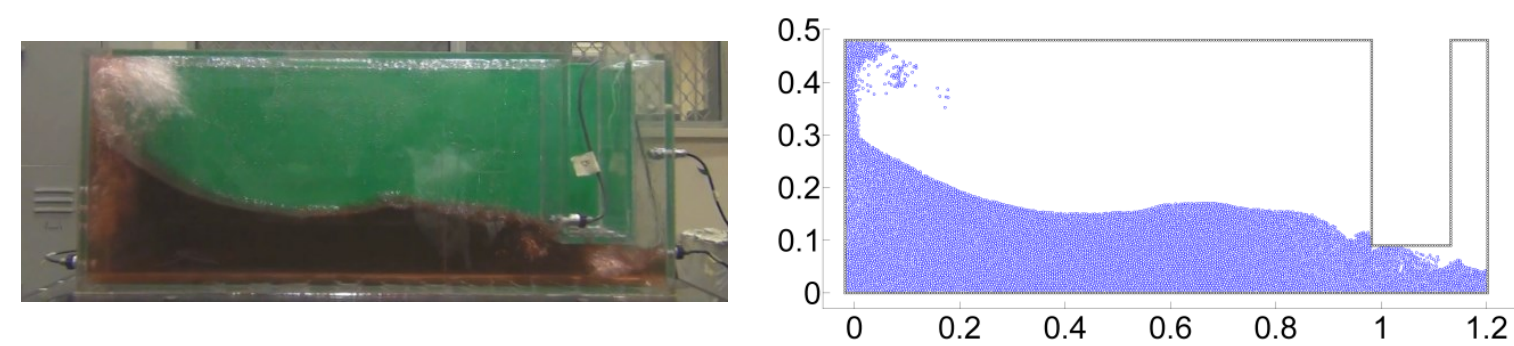

$$
t=7.56 \mathrm{~s}
$$

Figure 20. Wave profiles of sloshing without enclosed air pocket: experimental result and 1P-CPM simulation 


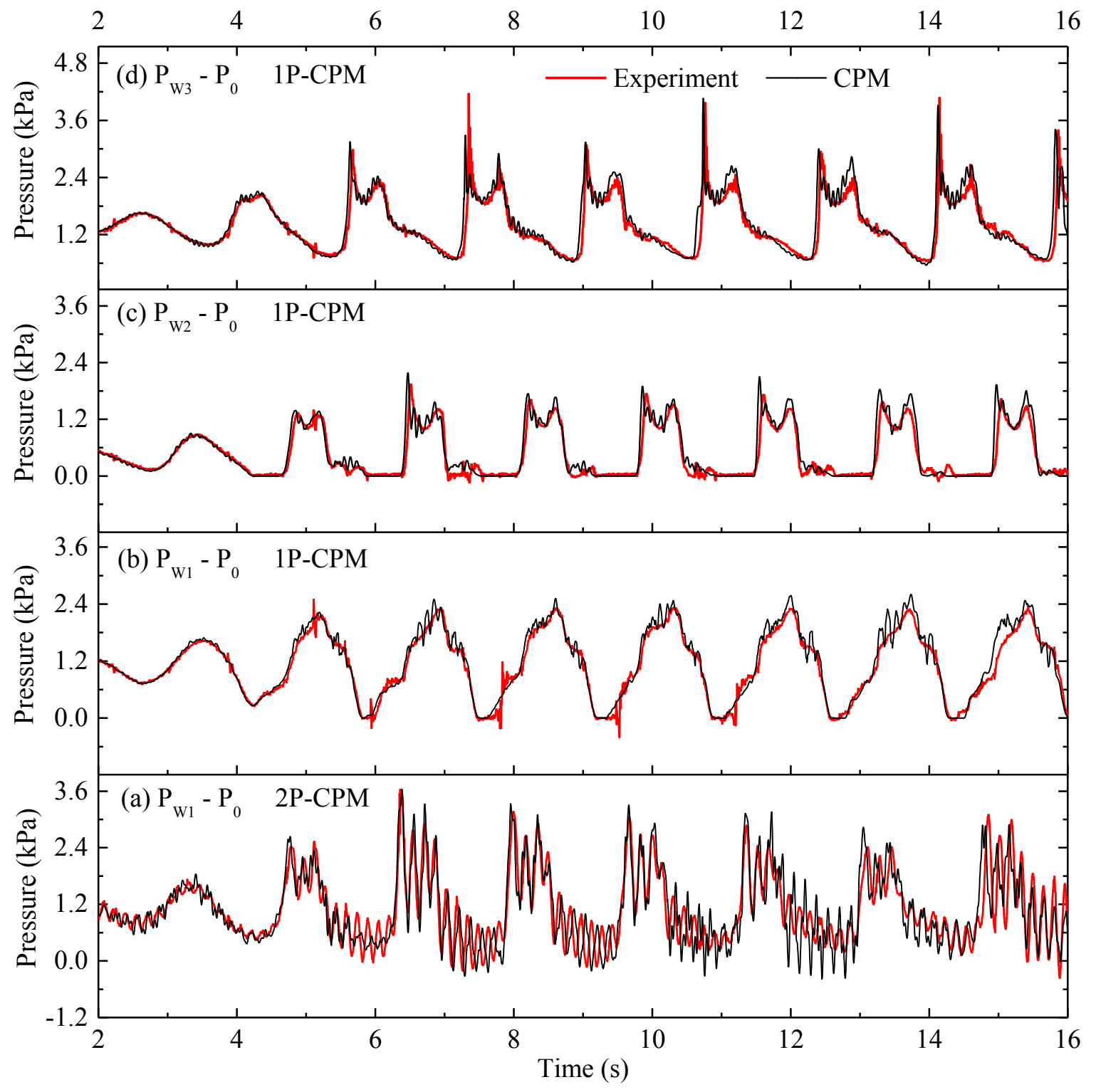

Figure 21. 1P-CPM results of water pressures at Point $\mathrm{P}_{\mathrm{w} 1}, \mathrm{P}_{\mathrm{W} 2}$ and $\mathrm{P}_{\mathrm{W} 3}$ in comparison with experimental results (without enclosed air pocket) and 2P-CPM result 


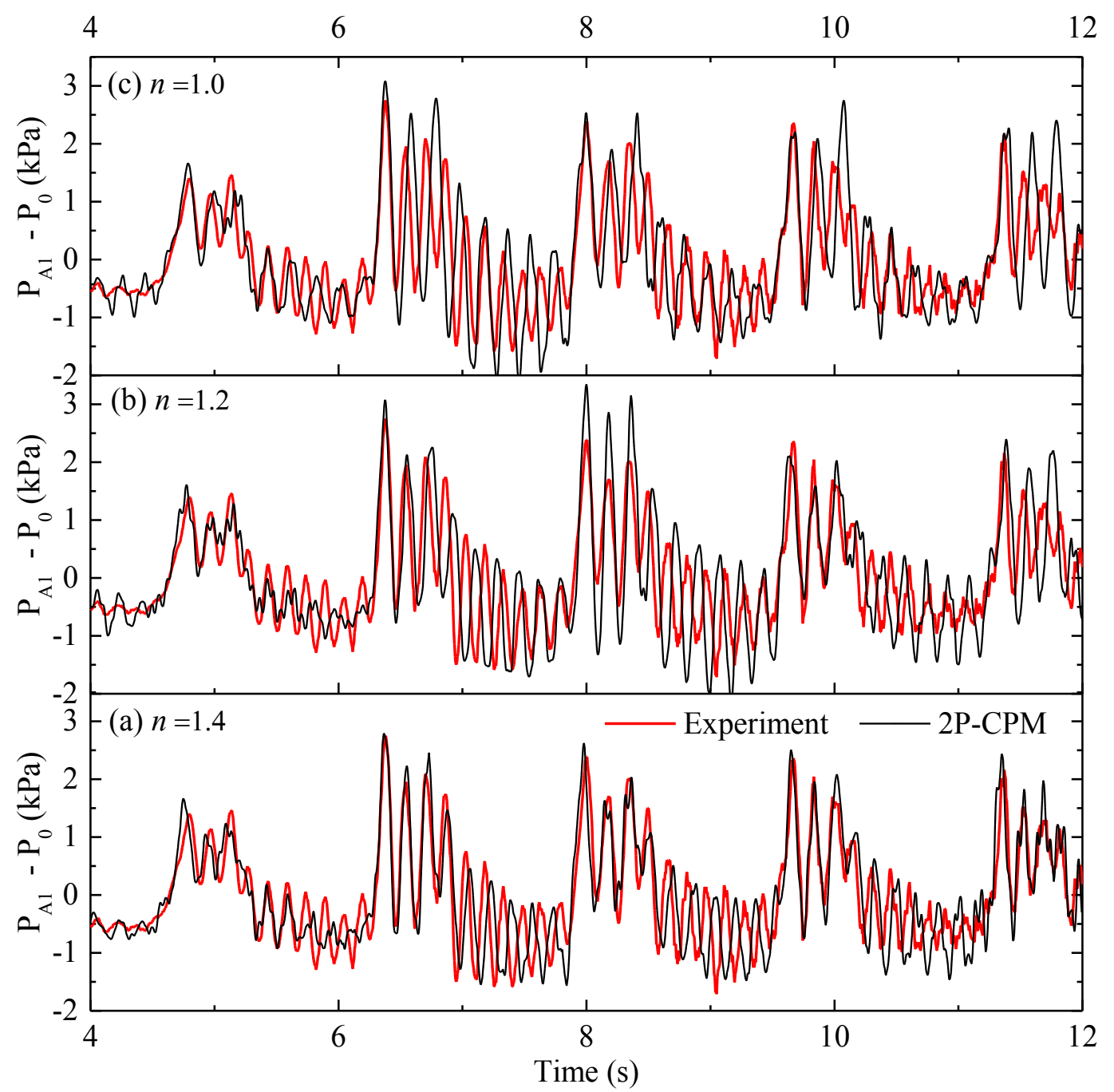

Figure 22. Air pressures at $\mathrm{P}_{\mathrm{A} 1}$ simulated by $2 \mathrm{P}-\mathrm{CPM}$ with polytropic index $n=1.4,1.2$ and 1.0 in comparison with experimental result 